\title{
Electroweak legacy of the LHC run II
}

\author{
Eduardo da Silva Almeida ${ }^{1,{ }^{*}}$ Alexandre Alves, ${ }^{2, \dagger}$ Oscar J. P. Éboli@, ${ }^{1, \$}$ and M. C. Gonzalez-Garcia ${ }^{3,4,5}$ \\ ${ }^{1}$ Instituto de Física, Universidade de São Paulo, Rua do Matão 1371, 05508-090 São Paulo, Brazil \\ ${ }^{2}$ Departamento de Física, Universidade Federal de São Paulo, UNIFESP, Diadema, São Paulo, Brazil \\ ${ }^{3}$ Departament de Fisica Quantica i Astrofisica and Institut de Ciencies del Cosmos, \\ Universitat de Barcelona, Diagonal 647, E-08028 Barcelona, Spain \\ ${ }^{4}$ Institució Catalana de Recerca i Estudis Avancats (ICREA) Passeig de Lluís Companys 23, \\ 08010 Barcelona, Spain \\ ${ }^{5}$ C. N. Yang Institute for Theoretical Physics, Stony Brook University, \\ Stony Brook, New York 11794-3849, USA
}

(Received 30 August 2021; revised 29 November 2021; accepted 6 December 2021; published 12 January 2022)

\begin{abstract}
We present a comprehensive study of the electroweak interactions using the available Higgs and electroweak diboson production results from LHC runs 1 and 2 as well as the electroweak precision data in terms of the dimension-six operators. Under the assumption that no new tree-level sources of flavor violation or violation of the universality of the weak current are introduced, the analysis involves 21 operators. We assess the impact of the data on kinematic distributions for the Higgs production at the LHC by comparing the results obtained by including the simplified template cross-section data with those in which only total Higgs signal strengths are considered. We also compare the results obtained when including the dimension-six anomalous contributions to order $1 / \Lambda^{2}$ and to order $1 / \Lambda^{4}$. As an illustration of the LHC potential to indirectly learn about specific forms of new physics, we adapt the analysis to constrain the parameter space for a few simple extensions of the standard model which generate a subset of the dimension-six operators at tree level.
\end{abstract}

DOI: 10.1103/PhysRevD.105.013006

\section{INTRODUCTION}

The large integrated luminosity accumulated by ATLAS and CMS during run 2 of the Large Hadron Collider (LHC) extended the reach of direct searches for new physics. It also allowed for studying some processes in more detaile.g., differential distributions are available for the Higgs production, or new channels were employed to explore the self-interactions of the electroweak gauge bosons. The outcome of the direct searches has been negative so far: no new states were observed. The most straightforward interpretation of this result points to a higher-thankinematically-accessible new physics scale. This makes the framework of effective Lagrangians [1-3] the obvious tool to search for indirect signals of (or limits on) new physics.

\footnotetext{
*eduardo.silva.almeida@usp.br

aalves@unifesp.br

*eboli@if.usp.br

§maria.gonzalez-garcia@stonybrook.edu
}

Published by the American Physical Society under the terms of the Creative Commons Attribution 4.0 International license. Further distribution of this work must maintain attribution to the author(s) and the published article's title, journal citation, and DOI. Funded by SCOAP.
The effective Lagrangian approach is suited for modelindependent analyses, since it is based exclusively on the low-energy-accessible states and symmetries. Assuming that the scalar particle observed in 2012 [4,5] belongs to an electroweak doublet, we can realize the $S U(2)_{L} \otimes U(1)_{Y}$ symmetry linearly. The resulting model is the so-called standard-model effective field theory (SMEFT). In this framework, dimension-six operators are the ones that can contribute to the LHC physics at the lowest order. But even limiting the effective Lagrangian to dimension six, the number of operators which can give signals at the LHC is large, stressing the relevance of the new kinematic information and the new observed channels.

In this work, we focus on the part of the dimension-six Lagrangian that modifies the electroweak interactions. Under the assumption of no new tree-level sources of flavor violation or violation of universality of the weak current, 21 dimension-six operators are relevant, and we introduce in Sec. II our choice of operator basis. Their Wilson coefficients parametrize our ignorance of the specific form of the new physics, and to obtain the strongest possible bounds in this general scenario, one must perform global analyses using all available experimental information. Here, we take into account the electroweak precision data (EWPD) and the data on electroweak diboson and 
Higgs productions at the LHC (see Sec. II). Recently, ATLAS and CMS released information on the kinematic distributions for the Higgs in the form of simplified template cross sections (STXSs) [6,7]. In order to understand the impact of such distributions with the present integrated luminosity, we have performed our studies using the STXS data as well as the Higgs results in the form of total signal strength (SS).

In a bottom-up approach, all Wilson coefficients are treated as free parameters. But, in a realistic UV completion, the Wilson coefficients are determined by the highenergy physics and might be correlated. In order to illustrate the importance of these correlations, we also obtain constraints on simple extensions of the standard model (SM) that give rise to dimension-six operators at tree level. We consider extensions with only one single particle added to the spectrum and two Higgs doublet models which we introduce in Sec. II A.

The results stemming from our global analyses, presented in Secs. IV and VI, show no statistically significant source of tension with the SM. We stress the complementarity of the different datasets in reaching this conclusion. In particular, we find that the LHC integrated luminosity is large enough to allow for non-negligible tightening of the bounds on some of the Wilson coefficients already constrained by the EWPD. Moreover, we show that the use of Higgs data is important to limit departures from the SM predictions for the triple electroweak gauge couplings (TGCs) due to the correlation introduced by the linear realization of the SM gauge symmetry in the effective operators.

Our analyses were conducted using the theoretical predictions linear [i.e., $\mathcal{O}\left(\Lambda^{-2}\right)$ ] in the Wilson coefficients, as well as including the quadratic $\left[\mathcal{O}\left(\Lambda^{-4}\right)\right]$ terms with the aim of estimating the uncertainties associated with the expansion in power of the high scale $\Lambda$. When working up to order $\Lambda^{-4}$, a set of (quasi)degenerate solutions appear associated with the flipping of the sign of some Higgs coupling. They lead to disconnected allowed ranges for the corresponding Wilson coefficients which do not contain the SM. Our analysis shows how Higgs kinematic distributions not only reduce the allowed SM-connected solutions, but also help in resolving some of these degeneracies, ruling out the non-SM-connected solutions for some of the operators. Generically, we find that, when focusing on SM-connected ranges of parameters, the precision on the bounds derived at $\mathcal{O}\left(\Lambda^{-2}\right)$ and at $\mathcal{O}\left(\Lambda^{-4}\right)$ become comparable for most operators. Quantitatively, our results are summarized in Table VI and Figs. 12 and 13.

At present, the comprehensive analysis of collider results in the framework of the SMEFT is in the hands of phenomenologists (see Refs. [8-13] for the most recent analyses). This article adds to this literature by including the most updated datasets and by the different working hypothesis employed. In brief, some of the cited analyses make a detailed study of flavor effects; however, they do not take into account the Higgs kinematic distribution in the STXS format; see, for instance, Refs. [8,10,11]. From the point of view of data samples included in the analysis, Refs. $[12,13]$ are the most complete, also including top results. But respecting Higgs analysis, they did not include the full updated ATLAS and CMS STXS of run 2 Higgs data, which we consider here. This allows us to quantify the present impact of the Higgs kinematic distribution data by contrasting the bounds obtained using STXS with those found using only the signal-strength data. In respect to the working hypothesis, we perform our analyses using the Hagiwara, Ishihara, Szalapski, and Zeppenfeld (HISZ) basis [14,15], which does not exhibit blind directions in the EWPD, in contrast with the Warsaw basis [16] employed in Refs. [8-13]; see Sec. II. Finally, as mentioned above, we perform our analysis both at $\mathcal{O}\left(\Lambda^{-2}\right)$ and at $\mathcal{O}\left(\Lambda^{-4}\right)$ as a way to quantitatively address the importance of higher-order terms and the use of EFT within its range of validity.

\section{THEORETICAL FRAMEWORK}

In order to describe the effects of new physics only directly accessible at a higher energy scale $\Lambda$, we supplement the SM with higher-dimension operators:

$$
\mathcal{L}_{\text {eff }}=\mathcal{L}_{\mathrm{SM}}+\sum_{n>4, j} \frac{f_{n, j}}{\Lambda^{n-4}} \mathcal{O}_{n, j},
$$

where the $\mathrm{SM} S U(3)_{C} \otimes S U(2)_{L} \otimes U(1)_{Y}$ gauge symmetry is realized linearly in the $\mathcal{O}_{n, j}$ operators. Neutrino physics strongly constrains the dimension-five operator [17]; therefore, dimension-six operators are those with the lowest dimension that contribute to processes at the LHC. For the sake of simplicity, we focus on dimension-six operators which conserve $C$ and $P$ (besides total lepton and baryon number). It is well known that there are 59 independent dimension-six operators [16], up to flavor and Hermitian conjugation. Here, we focus on those that modify the electroweak interactions impacting precision electroweak data, TGCs, and Higgs physics. For our analysis, 21 independent dimension-six operators are relevant, and we list our choice of basis in Tables I and II.

In particular, for the pure bosonic operators, we use the HISZ basis [14,15] (see Table I). $\Phi$ stands for the SM Higgs doublet, and we have defined $\hat{B}_{\mu \nu} \equiv i\left(g^{\prime} / 2\right) B_{\mu \nu}$ and $\hat{W}_{\mu \nu} \equiv$ $i(g / 2) \sigma^{a} W_{\mu \nu}^{a}$, with $g$ and $g^{\prime}$ being the $S U(2)_{L}$ and $U(1)_{Y}$ gauge couplings, respectively. In respect to the main effect of these operators in the observables, the $\mathcal{O}_{B W}$ and $\mathcal{O}_{\Phi, 1}$ operators are ubiquitous: they modify the electroweak gauge boson couplings among themselves and the couplings to the Higgs boson and fermions. The operator $\mathcal{O}_{\Phi, 2}$ rescales all the SM Higgs couplings. $\mathcal{O}_{B}, \mathcal{O}_{W}$, and $\mathcal{O}_{W W W}$ 
TABLE I. Dimension-six operators containing only bosonic fields that we considered in our analyses. The notation is given in the text.

\begin{tabular}{lcc}
\hline \hline $\mathcal{O}_{B W}=\Phi^{\dagger} \hat{B}_{\mu \nu} \hat{W}^{\mu \nu} \Phi$ & $\mathcal{O}_{\Phi, 1}=\left(D_{\mu} \Phi\right)^{\dagger} \Phi \Phi^{\dagger}\left(D^{\mu} \Phi\right)$ & $\mathcal{O}_{\Phi, 2}=\frac{1}{2} \partial^{\mu}\left(\Phi^{\dagger} \Phi\right) \partial_{\mu}\left(\Phi^{\dagger} \Phi\right)$ \\
$\mathcal{O}_{B}=\left(D_{\mu} \Phi\right)^{\dagger} \hat{B}^{\mu \nu}\left(D_{\nu} \Phi\right)$ & $\mathcal{O}_{W}=\left(D_{\mu} \Phi\right)^{\dagger} \hat{W}^{\mu \nu}\left(D_{\nu} \Phi\right)$ & $\mathcal{O}_{W W W}=\operatorname{Tr}\left[\hat{W}_{\mu}^{\nu} \hat{W}_{\nu}^{\rho} \hat{W}_{\rho}^{\mu}\right]$ \\
$\mathcal{O}_{G G}=\Phi^{\dagger} \Phi G_{\mu \nu}^{a} G^{a \mu \nu}$ & $\mathcal{O}_{B B}=\Phi^{\dagger} \hat{B}_{\mu \nu} \hat{B}^{\mu \nu} \Phi$ & $\mathcal{O}_{W W}=\Phi^{\dagger} \hat{W}_{\mu \nu} \hat{W}^{\mu \nu} \Phi$ \\
\hline \hline
\end{tabular}

TABLE II. Dimension-six operators containing fermionic fields that we considered in our analyses. The notation is given in the text.

\begin{tabular}{lcc}
\hline \hline $\mathcal{O}_{\Phi Q}^{(1)}=\Phi^{\dagger}\left(i \stackrel{\leftrightarrow}{D_{\mu}} \Phi\right)\left(\bar{Q} \gamma^{\mu} Q\right)$ & $\mathcal{O}_{\Phi Q}^{(3)}=\Phi^{\dagger}\left(i \stackrel{\leftrightarrow}{a} D_{\mu} \Phi\right)\left(\bar{Q}^{\mu} \gamma_{a} Q\right)$ & $\mathcal{O}_{\Phi u}^{(1)}=\Phi^{\dagger}\left(i \stackrel{\leftrightarrow}{D_{\mu}} \Phi\right)\left(\bar{u}_{R} \gamma^{\mu} u_{R}\right)$ \\
$\mathcal{O}_{\Phi d}^{(1)}=\Phi^{\dagger}\left(i \stackrel{\leftrightarrow}{D}_{\mu} \Phi\right)\left(\bar{d}_{R} \gamma^{\mu} d_{R}\right)$ & $\mathcal{O}_{\Phi u d}^{(1)}=\tilde{\Phi}^{\dagger}\left(i \stackrel{\leftrightarrow}{D_{\mu}} \Phi\right)\left(\bar{u}_{R} \gamma^{\mu} d_{R}\right)$ & $\mathcal{O}_{\Phi e}^{(1)}=\Phi^{\dagger}\left(i \stackrel{D}{D}_{\mu} \Phi\right)\left(\bar{e}_{R} \gamma^{\mu} e_{R}\right)$ \\
$\mathcal{O}_{t G}=\left(\bar{Q}_{3} \sigma^{\mu \nu} \frac{\lambda^{a}}{2} u_{3}\right) \tilde{\Phi} G_{\mu \nu}^{a}$ & & \\
$\mathcal{O}_{e \Phi, i i}=\left(\Phi^{\dagger} \Phi\right)\left(\bar{L}_{i} \Phi e_{R, i}\right) i=2,3$ & $\mathcal{O}_{u \Phi, 33}=\left(\Phi^{\dagger} \Phi\right)\left(\bar{Q}_{3} \tilde{\Phi} u_{R, 3}\right)$ & $\mathcal{O}_{d \Phi, 33}=\left(\Phi^{\dagger} \Phi\right)\left(\bar{Q}_{3} \Phi d_{R, 3}\right)$ \\
$\mathcal{O}_{L L L L}=\left(\bar{L} \gamma^{\mu} L\right)\left(\bar{L} \gamma^{\mu} L\right)$ & & \\
\hline \hline
\end{tabular}

contribute to triple gauge couplings, while $\mathcal{O}_{B}, \mathcal{O}_{W}, \mathcal{O}_{B B}$, $\mathcal{O}_{W W}$, and $\mathcal{O}_{G G}$ affect the Higgs couplings to gauge bosons.

We notice that our choice of operators differs from the independent basis choice in Ref. [16] and from that employed in other analyses (see Refs. [8-11,13,18-20] for some recent examples and references therein). Here, we keep the operators $\mathcal{O}_{B}$ and $\mathcal{O}_{W}$, and we remove instead some operators involving fermions.

The independent set of fermionic operators employed in our analysis is presented in Table II, where the lepton (quark) doublet is denoted by $L(Q)$ and $f_{R}$ are the $S U(2)_{L}$ singlet fermions. When including a subindex, it refers to the fermionic generation. When no subindex is included, the operator is the same for three generations. In addition, we define $\Phi^{\dagger} \stackrel{\leftrightarrow}{D}_{\mu} \Phi=\Phi^{\dagger} D_{\mu} \Phi-\left(D_{\mu} \Phi\right)^{\dagger} \Phi$ and $\Phi^{\dagger} \stackrel{\leftrightarrow}{D}_{\mu}^{a} \Phi=$ $\Phi^{\dagger} T^{a} D_{\mu} \Phi-\left(D_{\mu} \Phi\right)^{\dagger} T^{a} \Phi$ with $T^{a}=\sigma^{a} / 2$, with $\sigma^{a}$ being the Pauli matrices. The Gell-Mann matrices ${ }^{1}$ are represented by $\lambda^{a}$. In selecting the operators in Table II, we have assumed no family mixing in order to prevent the generation of too-large flavor violation [22,23], and, for the sake of simplicity, we have considered the operators involving couplings to gauge bosons to be generation independent. With these hypotheses, the operators involving lepton doublets $\mathcal{O}_{\Phi L}^{(1)}$ and $\mathcal{O}_{\Phi L}^{(3)}$ are removed from our basis [24], employing the freedom associated with the use of equations of motion [25-28]. This, as mentioned above, differs from the choice of basis in Ref. [16], in which these lepton doublet operators are kept in the exchange of the bosonic operators $\mathcal{O}_{B}$ and $\mathcal{O}_{W}$. The advantage of our choice is that it avoids the existence of blind directions $[29,30]$ in

\footnotetext{
${ }^{1}$ We did not considered six dipole operators whose interference with the SM contributions vanishes for EWPD observables. As shown in Ref. [21], including those additional operators would have no impact on the determination of the operators considered here.
}

the EWPD analyses so that the results of EWPD can be shown independently. It also allows for better discrimination of the impact of the LHC data on the precise determination of the fermion-gauge couplings versus gauge boson self-couplings.

In summary, the operators in the first two lines of Table II modify the fermion couplings to electroweak gauge bosons and the Higgs. And we notice that with our choice of basis, the only operator that modifies the $Z$ coupling to leptons is $\mathcal{O}_{\Phi e}^{(1)}$. On the other hand, the operator in the third line is the only one in our analyses affecting the fermion-gluon coupling, and it was included because it impacts the Higgs production via gluon fusion. The operators modifying the Yukawa interactions are presented in the fourth line, while the last one contains the only four-fermion operator that takes part in the determination of the Fermi constant.

Altogether, eight operators contribute at $\mathcal{O}\left(\Lambda^{-2}\right)$ at tree level to the EWPD observables [31], and the relevant part of the effective Lagrangian reads

$$
\begin{aligned}
\Delta \mathcal{L}_{\mathrm{eff}}^{\mathrm{EWPD}}= & \frac{f_{\Phi Q}^{(1)}}{\Lambda^{2}} \mathcal{O}_{\Phi Q}^{(1)}+\frac{f_{\Phi Q}^{(3)}}{\Lambda^{2}} \mathcal{O}_{\Phi Q}^{(3)}+\frac{f_{\Phi u}^{(1)}}{\Lambda^{2}} \mathcal{O}_{\Phi u}^{(1)}+\frac{f_{\Phi d}^{(1)}}{\Lambda^{2}} \mathcal{O}_{\Phi d}^{(1)} \\
& +\frac{f_{\Phi e}^{(1)}}{\Lambda^{2}} \mathcal{O}_{\Phi e}^{(1)}+\frac{f_{B W}}{\Lambda^{2}} \mathcal{O}_{B W}+\frac{f_{\Phi, 1}}{\Lambda^{2}} \mathcal{O}_{\Phi, 1} \\
& +2 \frac{f_{L L L L}}{\Lambda^{2}} \mathcal{O}_{L L L L} .
\end{aligned}
$$

The production of electroweak gauge boson pairs $W^{+} W^{-}, W^{ \pm} Z$, and $W^{ \pm} \gamma$ (from here on, EWDBD) receives contributions from dimension-six operators that modify the electroweak gauge boson couplings to fermions, as well as the triple gauge couplings. It is interesting to notice that dimension-six operators do not give rise to anomalous TGCs among neutral gauge bosons, which appear only at 
dimension eight [32]. The part of the effective Lagrangian that contributes to EWDBD, in addition to that participating in the EWPD analysis, is

$$
\begin{aligned}
\Delta \mathcal{L}_{\text {eff }}^{\mathrm{TGC}}= & \frac{f_{W W W}}{\Lambda^{2}} \mathcal{O}_{W W W}+\frac{f_{W}}{\Lambda^{2}} \mathcal{O}_{W}+\frac{f_{B}}{\Lambda^{2}} \mathcal{O}_{B} \\
& +\frac{f_{\text {Фud }}^{(1)}}{\Lambda^{2}}\left(\mathcal{O}_{\Phi u d}^{(1)}+\text { H.c. }\right) .
\end{aligned}
$$

The last operator modifies just the right-handed $W$ couplings to quarks, and consequently, it does not contribute to the EWPD observables at $\mathcal{O}\left(\Lambda^{-2}\right)$.

Dimension-six operators modify the Higgs production and decay through changes in its couplings to fermions and gauge bosons. We parametrize the contributions exclusive to the Higgs physics as

$$
\begin{aligned}
\Delta \mathcal{L}_{\mathrm{eff}}^{\mathrm{H}}= & \frac{f_{\mu} m_{\mu}}{\Lambda^{2} v} \mathcal{O}_{e \Phi, 22}+\frac{f_{\tau} m_{\tau}}{\Lambda^{2} v} \mathcal{O}_{e \Phi, 33}+\frac{f_{b} m_{b}}{\Lambda^{2} v} \mathcal{O}_{d \Phi, 33} \\
& +\frac{f_{t} m_{t}}{\Lambda^{2} v} \mathcal{O}_{u \Phi, 33}+\text { H.c. } \\
& -\frac{\alpha_{s}}{8 \pi} \frac{f_{G G}}{\Lambda^{2}} \mathcal{O}_{G G}+\frac{f_{B B}}{\Lambda^{2}} \mathcal{O}_{B B}+\frac{f_{W W}}{\Lambda^{2}} \mathcal{O}_{W W} \\
& +\frac{f_{\Phi, 2}}{\Lambda^{2}} \mathcal{O}_{\Phi, 2}+\frac{f_{t G}}{\Lambda^{2}} \mathcal{O}_{t G} .
\end{aligned}
$$

In summary, we consider the total effective Lagrangian:

$$
\mathcal{L}_{\text {eff }}=\mathcal{L}_{\mathrm{SM}}+\Delta \mathcal{L}_{\text {eff }}^{\mathrm{EWPD}}+\Delta \mathcal{L}_{\text {eff }}^{\mathrm{TGC}}+\Delta \mathcal{L}_{\text {eff }}^{\mathrm{H}} .
$$

For a complete list of vertices generated by Eq. (2.5), see Ref. [33]. The explicit form of the couplings and the different Lorentz structures generated can be found, for example, in Refs. [24,34-37], to which we refer the reader for details.

As we will see in the following section, at present there is enough experimental information to individually bound the 21 Wilson coefficients. But it is important to notice that some Wilson coefficients can lead to a sign change of Higgs couplings with respect to the SM predictions, consequently leading to discrete (quasi)degeneracies in the global analyses. For instance, the modification of the coefficient of the $H W_{\mu}^{+} W^{-\mu}$ vertex is

$$
\left(\frac{g^{2} v}{2}\right)\left[1-\frac{v^{2}}{4}\left(\frac{f_{\Phi, 1}}{\Lambda^{2}}+2 \frac{f_{\Phi, 2}}{\Lambda^{2}}\right)\right] .
$$

Since $f_{\Phi, 1} / \Lambda^{2}$ is strongly bounded by EWPD, it is only possible to have a degeneracy with the SM results for both $f_{\Phi, 2} / \Lambda^{2}=0$ and $f_{\Phi, 2} / \Lambda^{2} \sim 4 / v^{2} \sim 65 \mathrm{TeV}^{-2}$. These points in parameter space are also nearly degenerate for the vertex $H Z_{\mu} Z^{\mu}$.

In similar fashion, the anomalous interactions can also lead to Yukawa couplings of the order of the SM ones, but with a different sign, the coefficient of the $H \bar{f} f$ vertex is now

$$
-\frac{m_{f}}{v}\left[1-\frac{v^{2}}{4}\left(\frac{f_{\Phi, 1}}{\Lambda^{2}}+2 \frac{f_{\Phi, 2}}{\Lambda^{2}}+2 \sqrt{2} \frac{f_{f}}{\Lambda^{2}}\right)\right]
$$

where $f=\mu, \tau, b$, and $t$. Therefore, $f_{\Phi, 2} / \Lambda^{2}$ and $f_{f} / \Lambda^{2}$ can flip the sign of the Yukawa coupling. In combination with the twofold degeneracy $f_{\Phi, 2} / \Lambda^{2}$ in Eq. (2.6), there are $2 \times 2$ quasidegenerate SM-like solutions: two for $f_{f} / \Lambda^{2}=0$ and two with $f_{f} / \Lambda^{2} \pm 2 \sim \sqrt{2} / v^{2} \sim 45 \mathrm{TeV}^{-2}$ [33].

Another potential source of approximate degeneracies/ correlations is the effective photon-photon-Higgs coupling $H F_{\mu \nu} F^{\mu \nu}$, for which the dimension-six operators induce a tree-level contribution

$$
\begin{aligned}
A(\gamma \gamma \rightarrow H)= & A_{\mathrm{SM}}(\gamma \gamma \rightarrow H) \\
& +\frac{e^{2} v}{4} \frac{f_{W W}+f_{B B}-f_{B W}}{\Lambda^{2}},
\end{aligned}
$$

where $A_{\mathrm{SM}}(\gamma \gamma \rightarrow H) \simeq-8.25 \times 10^{-3} \mathrm{TeV}^{-1}$. First, taking into account the strong EWPD bounds on $f_{B W}$, this dependence leads to a strong anticorrelation between the allowed values of two remaining Wilson coefficients. That correlation is partly broken by the measurement of the effective photon- $Z$ coupling $H F_{\mu \nu} Z^{\mu \nu}$, which constrains a different combination of $f_{W W} / \Lambda^{2}, f_{B B} / \Lambda^{2}$, and $f_{B W} / \Lambda^{2}$. Furthermore, it is possible to find SM-like solutions for $\left(f_{W W}+f_{B B}\right) / \Lambda^{2} \sim 3 \mathrm{TeV}^{-2}$ with an inverted sign of the photon-photon-Higgs effective coupling.

The equivalent effect is also present in the gluon-gluonHiggs interaction. In the large-top-mass limit, the lowestorder amplitude is ${ }^{2}$

$$
\begin{aligned}
A(g g \rightarrow H)= & A_{\mathrm{SM}}(g g \rightarrow H)\left[1+\frac{3}{2} v^{2} \frac{f_{G G}}{\Lambda^{2}}\right. \\
& -\frac{v^{2}}{4}\left(\frac{f_{\Phi, 1}}{\Lambda^{2}}+2 \frac{f_{\Phi, 2}}{\Lambda^{2}}+2 \sqrt{2} \frac{f_{t}}{\Lambda^{2}}\right) \\
& \left.-\frac{\sqrt{2 \pi \alpha_{s}} v m_{t}^{3}}{m_{H}^{2}} \frac{f_{t G}}{\Lambda^{2}}\right] .
\end{aligned}
$$

It is important to notice that in most cases, the operators contributing to the Higgs vertices in Eqs. (2.6)-(2.9) involve different numbers of derivatives of the Higgs field. Consequently, we can anticipate the relevance of the data on kinematic distributions to resolve the vertex degeneracies and limit the correlations [38-40]—in particular, for gluon-gluon-Higgs, which contributes to the main Higgs production mechanism.

\footnotetext{
${ }^{2}$ Notice that we have defined the Wilson coefficient of the gluon-gluon-Higgs operator $f_{G G}$ in Eq. (2.4) to include a looplike suppression factor, so even if it gives a tree-level contribution to the amplitude, it can be factorized in this form in the large-topmass limit. We work here in a different convention than Refs. [24,33] and do not include in the definition of $\mathcal{O}_{G G}$ the effects of the anomalous operators that modify the couplings of the top quarks running in the loop.
} 


\section{A. Effective Lagrangians for simplified models}

Effective Lagrangians provide the framework for a bottom-up approach for the search for new physics through deviations of the SM predictions in a model-independent fashion. The disadvantage of this approach is that specific UV completions give rise, in general, to relations between the Wilson coefficients of different operators, which result in stronger constraints. To illustrate the potential of the effective Lagrangian framework, we consider some simple models whose matching at high energies are at tree level. For the sake of simplicity, we do not consider the running of the Wilson coefficients to the electroweak scale $[22,41,42]$. This renders the results independent of the matching scale and can lead to bounds which can either be slightly weaker or slightly stronger depending on the model [9].

(1) Extensions of the SM Higgs sector. The simplest model in this category is obtained by adding a singlet scalar $(S)$ which mixes with the SM Higgs with a mixing angle $\theta$. An interesting feature of the most general form of this model is that it can exhibit a strong first-order electroweak phase transition that can be addressed in complementary studies by colliders and gravitational wave experiments $[43,44]$. Here, however, we will focus on the case with a $Z_{2}$ symmetry $[9,45]$. At the high scale $\Lambda=M_{s}$, this model generates just one nonvanishing Wilson coefficient at tree level:

$$
\frac{f_{\Phi, 2}}{\Lambda^{2}}=\frac{\tan ^{2} \theta}{v^{2}}
$$

We also consider four classes of two-Higgs-doublet models (2HDMs) that satisfy the Weinberg-Glashow condition to avoid flavor-changing neutral currents [46]; these are labeled as 2HDM type I, 2HDM type II, 2HDM lepton specific, and 2HDM flipped (for further details, see Refs. [9,47,48]). The Wilson coefficients generated at tree level by these $2 \mathrm{HDMs}$ in the alignment limit $(|\cos (\beta-\alpha)| \ll 1)$ are $[9,48,49]$

$$
\begin{aligned}
& \frac{f_{b}}{\Lambda^{2}}=-\eta_{b} \frac{\cos (\beta-\alpha)}{\tan \beta} \frac{\sqrt{2}}{v^{2}}, \\
& \frac{f_{t}}{\Lambda^{2}}=-\eta_{t} \frac{\cos (\beta-\alpha)}{\tan \beta} \frac{\sqrt{2}}{v^{2}}, \\
& \frac{f_{\tau}}{\Lambda^{2}}=-\eta_{\tau} \frac{\cos (\beta-\alpha)}{\tan \beta} \frac{\sqrt{2}}{v^{2}},
\end{aligned}
$$

where $\tan \beta$ is the ratio between the Higgs doublet vacuum expectation values (VEVs) and $\alpha$ is the neutral Higgs mixing angle. The $\eta$ factor depends on the 2HDM type:

\begin{tabular}{lccc} 
2HDM & $\eta_{t}$ & $\eta_{b}$ & $\eta_{\tau}$ \\
\hline Type I & 1 & 1 & 1 \\
Type II & 1 & $-\tan ^{2} \beta$ & $-\tan ^{2} \beta$ \\
Lepton specific & 1 & 1 & $-\tan ^{2} \beta$ \\
Flipped & 1 & $-\tan ^{2} \beta$ & 1
\end{tabular}

(2) Simplified models [50] that contain the addition of one new (no-scalar) state. We consider five different charge assignments for the additional state as listed in Table III (see Ref. [51]). We also list in the third line the SM particles which couple to the new state. For consistency with our EFT assumptions, we assume equal couplings of the new state to the three

\begin{tabular}{|c|c|c|c|c|c|}
\hline $\begin{array}{l}\text { Additional state } \\
\text { Rep }\left(S U(3)_{c}, S U(2)_{L}, U(1)_{Y}\right) \\
\text { Couplings }\end{array}$ & $\begin{array}{l}\text { Vector } B \\
(1,1,0) \\
\text { Higgs }\end{array}$ & $\begin{array}{c}\text { Vector } B^{\prime} \\
(1,1,0) \\
\text { Higgs }+ \text { quarks }\end{array}$ & $\begin{array}{c}\text { Vector } W \\
(1,3,0) \\
\text { Higgs }+ \text { quarks }\end{array}$ & $\begin{array}{l}\text { Lepton } E \\
(1,1,-1) \\
\text { leptons }\end{array}$ & $\begin{array}{c}\text { Quark } U \\
(3,1,2 / 3) \\
\text { quarks }\end{array}$ \\
\hline$\overline{f_{\Phi, 1} / \Lambda^{2}}$ & -2 & -2 & $\ldots$ & $\ldots$ & $\ldots$ \\
\hline$f_{\Phi, 2} / \Lambda^{2}$ & 1 & 1 & $3 / 4$ & $\ldots$ & $\ldots$ \\
\hline$f_{\Phi, Q}^{(1)} / \Lambda^{2}$ & $\cdots$ & -1 & $\cdots$ & $\cdots$ & $1 / 4$ \\
\hline$f_{\Phi, Q}^{(3)} / \Lambda^{2}$ & $\cdots$ & $\ldots$ & $-1 / 4$ & $\ldots$ & $-1 / 4$ \\
\hline$f_{\Phi u}^{(1)} / \Lambda^{2}$ & $\cdots$ & -1 & $\cdots$ & $\cdots$ & $\cdots$ \\
\hline$f_{\Phi d}^{(1)} / \Lambda^{2}$ & $\cdots$ & -1 & $\ldots$ & $\cdots$ & $\ldots$ \\
\hline$f_{b} / \Lambda^{2}$ & $\ldots$ & $\ldots$ & $-\sqrt{2} / 4$ & $\ldots$ & $\ldots$ \\
\hline$f_{t} / \Lambda^{2}$ & $\cdots$ & $\cdots$ & $-\sqrt{2} / 4$ & $\cdots$ & $\sqrt{2} / 2$ \\
\hline$f_{\tau} / \Lambda^{2}$ & $\cdots$ & $\cdots$ & $\cdots$ & $\sqrt{2} / 2$ & $\cdots$ \\
\hline$f_{\mu} / \Lambda^{2}$ & $\cdots$ & $\ldots$ & $\cdots$ & $\sqrt{2} / 2$ & $\cdots$ \\
\hline$f_{\Phi \ell}^{(1)} / \Lambda^{2}$ & $\cdots$ & $\cdots$ & $\cdots$ & $-1 / 4$ & $\cdots$ \\
\hline$f_{\Phi \ell}^{(3)} / \Lambda^{2}$ & $\cdots$ & $\cdots$ & $\cdots$ & $-1 / 4$ & $\cdots$ \\
\hline
\end{tabular}

TABLE III. New particle content and Wilson coefficients generated by extending the SM with an extra state. All Wilson coefficients must be multiplied by $\chi^{2} / M^{2}$, where $\chi$ parametrizes the universal strength of the allowed couplings of the new state to the SM particles and $M$ is its mass. 
families. For simplicity, we also assume that the nonzero couplings of the new state to the SM particles have the same strength $\chi$.

With these assumptions, the Wilson coefficients generated at tree level by the new states are those listed in Table III. Notice that the existence of a heavy lepton $E(1,1)_{-1}$ generates two operators that were removed from our basis. Consequently, we have to rotate the result back to our basis using the fact that

$$
\begin{aligned}
\mathcal{O}_{\Phi \ell ; j j}^{(1)}= & \frac{8}{g^{\prime 2}} \mathcal{O}_{B}-2 \mathcal{O}_{\Phi, 2}+4 \mathcal{O}_{\Phi, 1}+\frac{4}{g^{\prime 2}} \mathcal{O}_{B B}+\frac{4}{g^{\prime 2}} \mathcal{O}_{B W} \\
& -2 \mathcal{O}_{\Phi e ; j j}+\frac{1}{3} \mathcal{O}_{\Phi Q ; j j}^{(1)}+\frac{4}{3} \mathcal{O}_{\Phi u ; j j}-\frac{2}{3} \mathcal{O}_{\Phi d ; j j}
\end{aligned}
$$

$$
\begin{aligned}
\mathcal{O}_{\Phi \ell ; j j}^{(3)}= & -\frac{8}{g^{2}} \mathcal{O}_{W}+6 \mathcal{O}_{\Phi, 2}-\frac{4}{g^{2}} \mathcal{O}_{W W}-\frac{4}{g^{2}} \mathcal{O}_{B W} \\
& -\mathcal{O}_{\Phi Q ; j j}^{(3)}-2\left[Y_{u}^{\dagger} \mathcal{O}_{u \Phi}+Y_{d}^{\dagger} \mathcal{O}_{d \Phi}+Y_{e}^{\dagger} \mathcal{O}_{e \Phi}\right],
\end{aligned}
$$

where $Y_{f}$ stands for the Yukawa coupling of the fermion $f$.

\section{ANALYSIS FRAMEWORK}

In order to study the Wilson coefficients of the dimension-six operators of the effective Lagrangian [Eq. (2.5)], we take into account the EWPD, diboson production, and Higgs data on signal strength and simplified template crosssection measurements. We perform the global analyses either using only the contributions that are linear on the Wilson coefficients $\left[\mathcal{O}\left(1 / \Lambda^{2}\right)\right]$, or including the quadratic terms $\left[\mathcal{O}\left(1 / \Lambda^{4}\right)\right]$ as well.
In the EWPD analysis, we study 15 observables, of which 12 are $Z$ observables [31]:

$\Gamma_{Z}, \quad \sigma_{h}^{0}, \quad \mathcal{A}_{\ell}\left(\tau^{\mathrm{pol}}\right), \quad R_{\ell}^{0}, \quad \mathcal{A}_{\ell}(\mathrm{SLD}), \quad A_{\mathrm{FB}}^{0, l}, \quad R_{c}^{0}$,

$R_{b}^{0}, \quad \mathcal{A}_{c}, \quad \mathcal{A}_{b}, \quad A_{\mathrm{FB}}^{0, c}, \quad$ and $A_{\mathrm{FB}}^{0, b}(\mathrm{SLD} / \mathrm{LEP}-\mathrm{I})$,

supplemented by three $W$ observables: he $W$ mass $\left(M_{W}\right)$ taken from Ref. [52], its width $\left(\Gamma_{W}\right)$ from LEP2/Tevatron [53], and the leptonic $W$ branching ratio $[\operatorname{Br}(W \rightarrow \ell \nu)]$ [52].

The first component of our global $\chi^{2}$ function is therefore suited for the EWPD data:

$\chi_{\mathrm{EWPD}}^{2}\left(f_{B W}, f_{\Phi, 1}, f_{\Phi, Q}^{(3)}, f_{\Phi, Q}^{(1)}, f_{\Phi, u}^{(1)}, f_{\Phi, d}^{(1)}, f_{\Phi, e}^{(1)}, f_{L L L L}\right)$.

In the EWPD analysis, we include the correlations among the above observables, as displayed in Ref. [31], while the SM predictions and uncertainties are extracted from Ref. [54]. For further details of this part of the statistical analysis, we refer the reader to Refs. [35,37].

Triple gauge boson couplings have already been studied in the production of electroweak gauge boson pairs at LEP2 [55], and in runs 1 and 2 of the LHC [56-67]. Here we analyze the most complete samples of $W^{+} W^{-}, W^{ \pm} Z, W^{ \pm} \gamma$, and $Z j j$. More specifically, the channels that we study and the kinematic distribution included in the analysis are listed in Table IV. Further details on the analysis of EWDBD from run 1 can be seen in Ref. [37]. Generically, for most data samples from the above publications, we extract the observed event rates in each bin $\left(N_{\mathrm{i}, \mathrm{d}}^{a}\right)$, as well as the background expectations $\left(N_{i, \mathrm{bck}}^{a}\right)$ and the SM predictions $\left(N_{i, \mathrm{sm}}^{a}\right)$ for each channel. An exception are the results for the $W^{ \pm} \gamma$ of CMS [65], and $Z j j$ in ATLAS [67], for which the experiments provide the results in the form of reconstructed differential cross sections.

In our analyses, we simulate the results in the $W^{+} W^{-}$, $W^{ \pm} Z, W^{ \pm} \gamma$, and $Z j j$ channels that receive contributions

\begin{tabular}{|c|c|c|c|c|}
\hline Channel $(a)$ & Distribution & Number of bins & Dataset & Integrated luminosity \\
\hline$W W \rightarrow \ell^{+} \ell^{\prime-}+E_{T}(0 j)$ & $p_{T}^{\text {leading,lepton }}$ & 3 & ATLAS $8 \mathrm{TeV}$, & $20.3 \mathrm{fb}^{-1}[56]$ \\
\hline$W W \rightarrow \ell^{+} \ell^{(\prime)}-+E_{T}^{\prime}(0 j)$ & $m_{\ell \ell^{(\prime)}}$ & 8 & CMS $8 \mathrm{TeV}$, & $19.4 \mathrm{fb}^{-1}[57]$ \\
\hline$W Z \rightarrow \ell^{+} \ell^{-} \ell^{(\prime) \pm}$ & $m_{T}^{W Z}$ & 6 & ATLAS $8 \mathrm{TeV}$, & $20.3 \mathrm{fb}^{-1}[58]$ \\
\hline$W Z \rightarrow \ell^{+} \ell^{-} \ell^{(\prime) \pm}+E_{T}$ & $Z$ candidate $p_{T}^{\ell \ell}$ & 10 & CMS $8 \mathrm{TeV}$, & $19.6 \mathrm{fb}^{-1}[59]$ \\
\hline$W W / W Z \rightarrow \ell \nu J$ & $p_{T}^{J}$ & 6 & ATLAS $8 \mathrm{TeV}$, & $20.2 \mathrm{fb}^{-1}[62]$ \\
\hline$W Z \rightarrow \ell^{+} \ell^{-} \ell^{\prime \pm}$ & $M(W Z)$ & 7 & CMS $13 \mathrm{TeV}$ & $137.2 \mathrm{fb}^{-1}[63]$ \\
\hline$W W \rightarrow \ell^{+} \ell^{(\prime)-}+0 / 1 j$ & $M\left(\ell^{+} \ell^{(\prime)-}\right)$ & 11 & CMS $13 \mathrm{TeV}$ & $35.9 \mathrm{fb}^{-1}[64]$ \\
\hline$W \gamma \rightarrow \ell \nu \gamma$ & $\frac{d^{2} \sigma}{d p_{T} d \phi}$ & 12 & CMS $13 \mathrm{TeV}$ & $137.1 \mathrm{fb}^{-1}[65]$ \\
\hline$W W \rightarrow e^{ \pm} \mu^{\mp}+E_{T}^{6}(0 j)$ & $m_{T}$ & $17(15)$ & ATLAS $13 \mathrm{TeV}$, & $36.1 \mathrm{fb}^{-1}[66]$ \\
\hline$W Z \rightarrow \ell^{+} \ell^{-} \ell^{(\prime) \pm}$ & $m_{T}^{W Z}$ & 6 & ATLAS $13 \mathrm{TeV}$, & $36.1 \mathrm{fb}^{-1}[61]$ \\
\hline$Z j j \rightarrow \ell^{+} \ell^{-} j j$ & $\frac{d \sigma}{d \phi}$ & 12 & ATLAS $13 \mathrm{TeV}$, & $139 \mathrm{fb}^{-1}[67]$ \\
\hline
\end{tabular}
from TGC and anomalous fermion pair couplings to gauge

TABLE IV. Diboson data from the LHC used to constrain the dimension-six operators. For the $W^{+} W^{-}$results from ATLAS run 2 [66], we combine the data from the last three bins into one to ensure Gaussianity. 
bosons using MadGraph5_aMC@NLO [68] with the UFO files for our effective Lagrangian generated with FeynRules [69,70]. We employ PYTHIA8 [71] to perform the parton shower and hadronization, while the fast detector simulation is carried out with DELPHES [72]. Jet analyses were performed using FASTJET [73].

In order to account for higher-order corrections and additional detector effects, we obtain the SM diboson cross section in the fiducial region defined by the experimental collaborations, requiring the same cuts and isolation criteria employed in the experimental studies, and we normalize our results bin by bin to the experimental collaboration predictions for the kinematic distributions under consideration. Then, we apply these correction factors to our simulated contributions to diboson distributions from the relevant dimension-six operators.

These predictions are statistically confronted with the data from LHC runs 1 and 2 by constructing a binned log-likelihood function based on the data contents of the different bins in the kinematic distribution of each channel. In addition to the statistical errors, we incorporate the systematic and theoretical uncertainties, adding them in quadrature and assuming some partial correlation among them, which we estimate with the information provided by the experiments. In particular, some of the experimental publications contain analysis of their EWDB data in the framework of some anomalous TGCs. In those cases, we can compare the allowed region of parameter space obtained with our constructed likelihood function for that data sample with that of the experiment, and we can use that comparison to fine-tune our treatment of the systematic uncertainties and correlations to match the results of the experimental analysis as closely as possible. Altogether, we build the function $\chi_{\text {EWDBD }}^{2}$, summing the $\chi^{2}$ constructed for the analysis of each sample in Table IV. Then, combining it with the EWPD bounds, we define

$$
\chi_{\mathrm{EWPD}+\mathrm{EWDBD}}^{2}\left(f_{B}, f_{W}, f_{W W W}, f_{B W}, f_{\Phi, 1}, f_{\Phi, Q}^{(3)}, f_{\Phi, Q}^{(1)}, f_{\Phi, u}^{(1)}, f_{\Phi, d}^{(1)}, f_{\Phi, u d}^{(1)}, f_{\Phi, e}^{(1)}, f_{L L L L}\right) .
$$

Respecting Higgs results in our studies, we use the available Higgs data depicted in Table V. Notice that Ref. [74] summarizes the ATLAS results on $H \rightarrow \gamma \gamma$ [75], $H \rightarrow 4 \ell$ [76], and $H \rightarrow b \bar{b}$ [77], and the detailed statistics information can be extracted from these references.

In order to assess the effects of including the kinematic information, we have performed two different analyses of the Higgs results. In the first one, we only include the data on the total signal strengths. We label that Analysis SS. This includes the results labeled as Analysis SS in Table V, which corresponds to 22 data points for run 1 and
$11($ ATLAS $)+23(\mathrm{CMS})=34$ data points for run 2 , for a total of 56 data points.

In the second analysis, labeled as Analysis STXS, we make use of all the available information on the kinematic distributions as provided in the form of simplified template cross sections. For those channels and luminosities for which the STXS data is not available, we use the corresponding data as the total signal strength. In this form, the STXS analysis includes the same 22 data points for run 1 and 45 (58) STXS points for the ATLAS (CMS) run 2, supplemented with the 7 (12) SS points for the channels for which no STXS data is available. So, in total, the STXS

TABLE V. Higgs data used to compute $\chi_{\text {Higgs }}^{2}$ In the column labeled "Data format," SS stands for total signal strength and STXS for simplified template cross section. The column marked "Analysis" specifies which of the two analyses include each dataset. In that column, SS labels the analysis which includes only total signal strengths for all channels, and STXS labels the analysis which includes instead the kinematic information in the form of simplified template cross sections for those channels for which it is available.

\begin{tabular}{|c|c|c|c|c|}
\hline Source & Data format & Analysis & Integrated luminosity $\left(\mathrm{fb}^{-1}\right)$ & Number of Data points \\
\hline ATLAS + CMS at $7 \& 8 \mathrm{TeV}$ [78] (Table 8, Fig. 27) & SS & SS \& STXS & $5 \& 20$ & $20+1$ \\
\hline ATLAS at $8 \mathrm{TeV}[79](\gamma Z)$ & SS & SS \& STXS & 20 & 1 \\
\hline ATLAS at $13 \mathrm{TeV}$ [80] (Figs. 7 and 20) & SS & SS & $36.1-139$ & 9 \\
\hline ATLAS at $13 \mathrm{TeV}[81](\gamma Z)$ & SS & SS \& STXS & 139 & 1 \\
\hline ATLAS at $13 \mathrm{TeV}[82]\left(\mu^{+} \mu^{-}\right)$ & SS & SS \& STXS & 139 & 1 \\
\hline ATLAS at $13 \mathrm{TeV}[74](\gamma \gamma, 4 \ell, b \bar{b})$ & STXS & STXS & 139 & 43 \\
\hline ATLAS at $13 \mathrm{TeV}$ [80] (Figs. 5 and 6) & SS & STXS & $36.1-139$ & 7 \\
\hline CMS at $13 \mathrm{TeV}$ [83] (Table 5) & SS & SS & $35.9-137$ & 23 \\
\hline $\mathrm{CMS}$ at $13 \mathrm{TeV}[84](\gamma \gamma)$ & STXS & STXS & 137 & 24 \\
\hline $\mathrm{CMS}$ at $13 \mathrm{TeV}[85](4 \ell)$ & STXS & STXS & 137 & 19 \\
\hline $\mathrm{CMS}$ at $13 \mathrm{TeV}[86]\left(\tau^{+} \tau^{-}\right)$ & STXS & STXS & 137 & 11 \\
\hline $\mathrm{CMS}$ at $13 \mathrm{TeV}[87]\left(W^{+} W^{-}\right)$ & STXS & STXS & 137 & 4 \\
\hline CMS at $13 \mathrm{TeV}$ [83] (Table 5) & SS & STXS & $35.9-137$ & 12 \\
\hline
\end{tabular}


analysis includes 144 data points. It is important to notice that the STXS results of ATLAS for different channels are all presented in Ref. [74], where a full correlation matrix between the different STXS bins for different channels is provided. On the contrary, the different STXS results from CMS are given in different publications Refs. [84-87] for different channels, and no correlations between bins for different channels are available. So, in our analysis, we have set those to zero.

We obtain the theoretical predictions for the Higgs production by gluon fusion in the channels tagged as STXS in Table V using MadGraph5_aMC@NLO [88] with the SMEFT@NLO UFO files [89]. Notice that we took into account not only the contribution to this process from $\mathcal{O}_{G G}$ but also those coming from $\mathcal{O}_{\Phi, 2}, \mathcal{O}_{u \Phi, 33}$, and $\mathcal{O}_{t G}$. Moreover, the STXS 1.2 classification was performed using RIVET [90].
The statistical comparison of our effective theory predictions with the LHC results including those two data samples is made by means of a $\chi^{2}$ analysis performed in the 21-dimensional statistical function and adding the information from EWPD and EWDBD. Furthermore, the operator $\mathcal{O}_{t G}$ contributes not only to the gluon-fusion Higgs production [91,92], but also to the top production [12,93-97], which provides independent information. We include such information on this operator in the form of a Gaussian bias built with the result of the full global fit to top-quark physics performed in Ref. [95]:

$$
\chi_{\text {bias,top }}^{2}\left(f_{t G}\right)=\frac{\left(\frac{f_{t G}}{\Lambda^{2}}-0.5\right)^{2}}{(0.22)^{2}} .
$$

Altogether, we define the $\chi^{2}$ for our global analysis as

$$
\begin{aligned}
\chi_{\text {Global SS(STXS) }}^{2}= & \chi_{\text {EWPD+EWDBD }}^{2}\left(f_{B}, f_{W}, f_{W W W}, f_{B W}, f_{\Phi, 1}, f_{\Phi, Q}^{(3)}, f_{\Phi, Q}^{(1)}, f_{\Phi, u}^{(1)}, f_{\Phi, d}^{(1)}, f_{\Phi, u d}^{(1)}, f_{\Phi, e}^{(1)}, f_{L L L L}\right) \\
& +\chi_{\text {HIGGSSS(STXS) }}^{2}\left(f_{B}, f_{W}, f_{B B}, f_{W W}, f_{B W}, f_{G G}, f_{\Phi, 1} f_{\Phi, 2}, f_{\Phi, Q}^{(3)}, f_{\Phi, Q}^{(1)}, f_{\Phi, u}^{(1)}, f_{\Phi, d}^{(1)}, f_{\Phi, u d}^{(1)}, f_{\Phi, e}^{(1)}, f_{t G}, f_{b}, f_{t}, f_{\tau}, f_{\mu}\right) \\
& +\chi_{\text {bias,top }}^{2}\left(f_{t G}\right) .
\end{aligned}
$$

\section{EFFECTIVE FIELD THEORY RESULTS}

Figures $1-8$ and 11 depict $\Delta \chi^{2}$ profiles for the dimension-six Wilson coefficients, where we have marginalized with respect to all undisplayed parameters. We show the results for four different combinations of datasets:

(1) EWPD: $\Delta \chi_{\text {EWPD }}^{2}$, which constrains the 8 coefficients in $\Delta \mathcal{L}_{\text {eff }}^{\text {EWPD }}$ [Eq. (2.2)]. They are given by the green lines in Fig. 1, which have been obtained by considering only the contributions to the observables that are $\mathcal{O}\left(\Lambda^{-2}\right)$; see Ref. [35]. We also assess the impact of relaxing the flavor universality hypothesis by considering a different coupling to the third family, whose result is displayed in Fig. 2.

(2) EWPD + EWDBD: $\Delta \chi_{\text {EWPD+EWDBD }}^{2}$, which limits the 12 coefficients in $\Delta \mathcal{L}_{\text {eff }}^{\text {EWP }}+\Delta \mathcal{L}_{\text {eff }}^{\text {TGC }}$ [Eqs. (2.2) and (2.3)]. In the EWDBD analysis, we consider contributions to the observables up to $\mathcal{O}\left(\Lambda^{-2}\right)$ and up to $\mathcal{O}\left(\Lambda^{-4}\right)$. Our results are displayed in Fig. 3.

(3) GLOBAL: $\Delta \chi_{\text {Global ANALYSIS }}^{2}$ [Eq. (3.5)], which constrains the 21 coefficients in $\mathcal{L}_{\text {eff }}$ in Eqs. (2.2)-(2.4). As described in the previous section, we consider two different sets of HIGGS data, one which includes only the total signal strengths, Analysis SS, and one which includes also the information on the kinematic distributions, Analysis STXS (see Table V). For each case we perform two analyses: one in which the theoretical predictions are expanded to $\mathcal{O}\left(\Lambda^{-2}\right)$, and another one including the predictions up to $\mathcal{O}\left(\Lambda^{-4}\right)$. Different projections of the corresponding $\Delta \chi_{\text {Global ANALYSIS }}^{2}$ are shown in Figs. 1-8 and 11 summarizes the results for the STXS analysis.

\section{A. Lessons from the EWPD}

We start our analysis by focusing on the dimension-six operators in Eq. (2.2) that directly impact EWPD. Our results are presented in Fig. 1. The bounds imposed by the EWPD analysis are represented by the green lines, while the limits coming from the global SS (STXS) analyses are given by the black (red) lines, including up to $\mathcal{O}\left(\Lambda^{-4}\right)$ and up to $\mathcal{O}\left(\Lambda^{-2}\right)$ terms as full and dashed lines, respectively. As is well known, EWPD leads to very stringent constraints on fermion-gauge interactions and on new oblique corrections to the gauge boson propagators. However, as illustrated in the figure, with the accumulated LHC statistics, EWDBD and Higgs results already contribute to tighten the EWPD bounds for some of the coefficients despite the larger 21-parameter space.

In the upper-left and upper-central panels of Fig. 1, we find the $\Delta \chi^{2}$ dependence on $f_{B W} / \Lambda^{2}$ and $f_{\Phi, 1} / \Lambda^{2}$, which correspond to the $S$ and $T$ oblique corrections, respectively. For these coefficients, the EWPD limits are only slightly improved by the global analysis including the STXS Higgs data when the theoretical predictions include the quadratic contributions of the Wilson coefficients.

Turning to the quark gauge couplings, the upper-right (middle-left) panel of Fig. 1 presents the $\Delta \chi^{2}$ dependence 

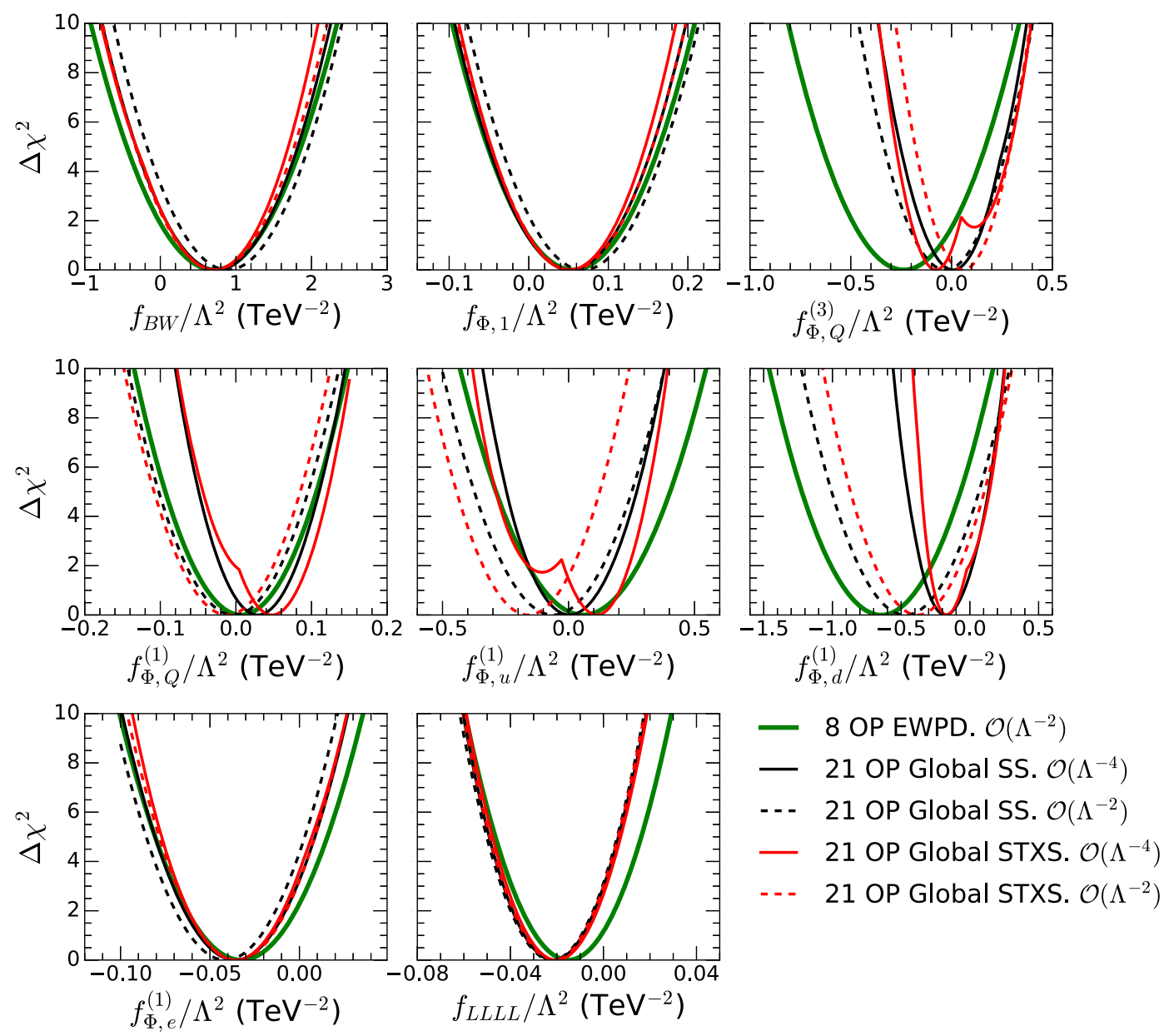

FIG. 1. $\Delta \chi^{2}$ as a function of the Wilson coefficients $f_{B W} / \Lambda^{2}, f_{\Phi, 1} / \Lambda^{2}, f_{\Phi, Q}^{(1)} / \Lambda^{2}, f_{\Phi, Q}^{(3)} / \Lambda^{2}, f_{\Phi, u}^{(1)} / \Lambda^{2}, f_{\Phi, d}^{(1)} / \Lambda^{2}, f_{\Phi, e}^{(1)} / \Lambda^{2}$, and $f_{L L L L} / \Lambda^{2}$, as indicated in the panels after marginalizing over the remaining fit parameters. The green solid line stands for the fit of the EWPD that constrains only eight of 21 Wilson coefficients in Eq. (2.5). The red solid (dashed) line represents the 21-parameter fit to the LHC run 1 and 2 data including the STXS Higgs data and working at order- $1 / \Lambda^{4}$ (order- $1 / \Lambda^{2}$ ) approximation. For comparison, we present the corresponding results for the global fit using the SS Higgs data (black solid and dashed lines).

on the coefficient $f_{\Phi, Q}^{(3)} / \Lambda^{2}\left(f_{\Phi, Q}^{(1)} / \Lambda^{2}\right)$, which modifies the couplings of left-handed quarks to $Z$ and $W$ (only $Z$ ) bosons. The middle-central and middle-right panels correspond to the dependences on $f_{\Phi, u}^{(1)} / \Lambda^{2}$ and $f_{\Phi, d}^{(1)} / \Lambda^{2}$, which give corrections to the $u_{R}$ and $d_{R}$ couplings to $Z$, respectively. The inclusion of the LHC observables leads to a small but not negligible improvement on the determination of $f_{\Phi, u}^{(1)} / \Lambda^{2}$ at linear order, while it only affects the EWPD bounds on $f_{\Phi, Q}^{(1)} / \Lambda^{2}$ when $\mathcal{O}\left(\Lambda^{-4}\right)$ terms are included in the LHC analysis. The largest effect of the combination with the LHC observables occurs for $f_{\Phi d}^{(3)} / \Lambda^{2}$ and $f_{\Phi, Q}^{(3)} / \Lambda^{2}$. In particular, the EWPD analysis favors nonvanishing values for $f_{\Phi d}^{(1)} / \Lambda^{2}$ at $2 \sigma$, a result driven by the $2.7 \sigma$ discrepancy between the observed $A_{\mathrm{FB}}^{0, b}$ and the SM. On the contrary, the inclusion of the LHC data gives rise to a shift towards zero of $f_{\Phi d}^{(1)} / \Lambda^{2}$, reducing the tension with the SM. This behavior was already observed in Refs. [33,37], but we find now that with the accumulated statistics, LHC also is able to provide relevant constraints when including the effect of dimension-six operators only at $\mathcal{O}\left(\Lambda^{-2}\right)$. As the LHC observables included are mostly sensitive to gauge couplings of the light quarks in the parton distribution functions, one expects that this result relies upon the assumption that the operators involving fermion couplings to gauge bosons are generation independent. To test this, we perform an analysis in which we drop this assumption for the operator $f_{\Phi d}^{(1)} / \Lambda^{2}$ and allow $f_{\Phi, d_{11}}^{(1)}=f_{\Phi, d_{22}}^{(1)} \neq f_{\Phi, d_{33}}^{(1)}$. The results are shown in Fig. 2, where we display the

\footnotetext{
${ }^{3}$ If one relaxes the assumption of generation-independent quark couplings, the EWPD cannot constrain all quark couplings, because there is not enough information in the observables considered to resolve the contributions of the two first generations. Furthermore, for the third generation of quarks, only $f_{\Phi, d_{33}}^{(1)}$ and the linear combination $4 f_{\Phi, Q_{33}}^{(1)}+f_{\Phi, Q_{33}}^{(3)}$ contribute independently to the $Z$ and $W$ observables.
} 

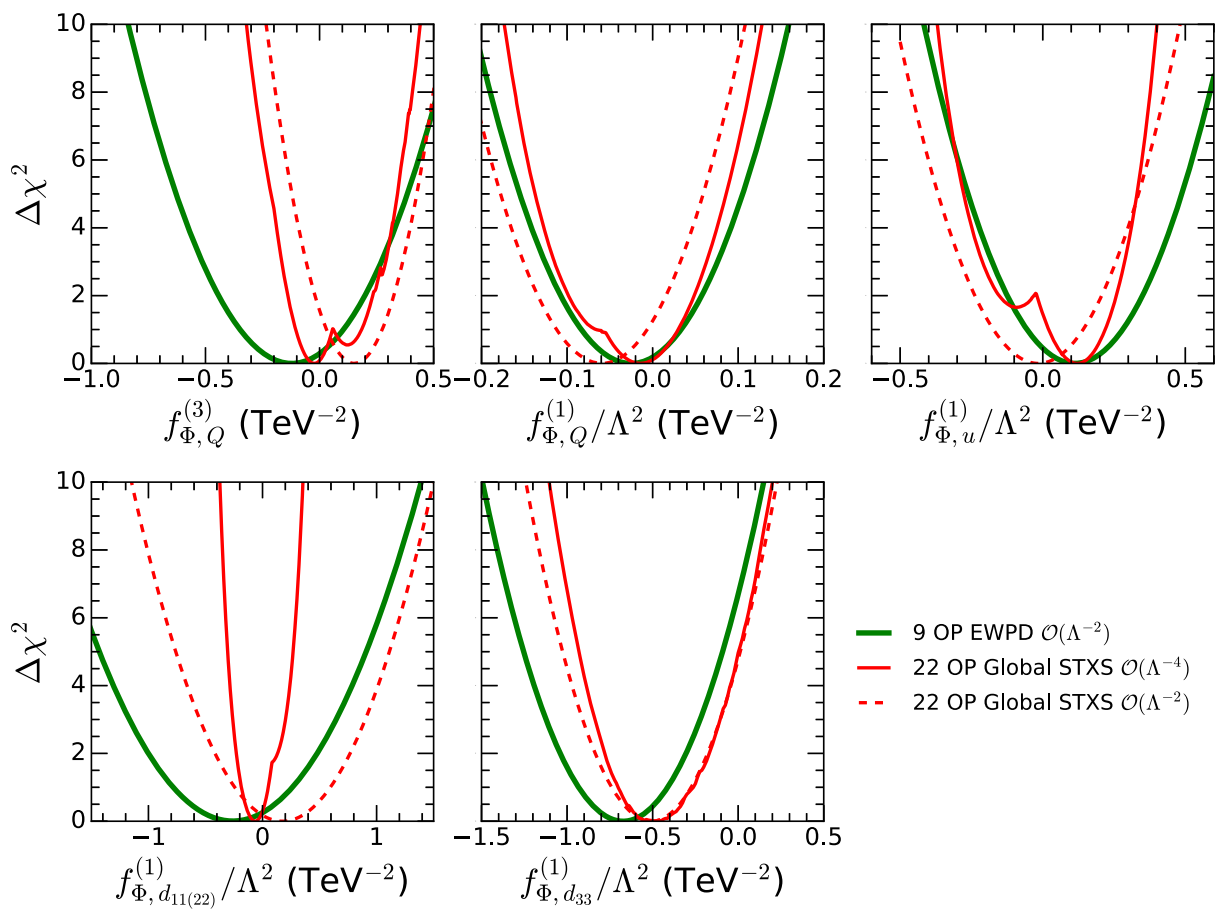

FIG. 2. $\Delta \chi^{2}$ as a function of the Wilson coefficients contributing to the gauge couplings of the quarks for nonuniversal right-handed quark coupling of the bottom quark; this is for $f_{\Phi, d_{1}}^{(1)}=f_{\Phi, d_{2}}^{(1)} \neq f_{\Phi, d_{3}}^{(1)}$. The green solid line stands for the fit of the EWPD that constrains only nine of 22 Wilson coefficients. The red solid (dashed) line represents the 21-parameter fit to the LHC run 1 and 2 data including the STXS Higgs data and working at order- $1 / \Lambda^{4}$ (order- $\left.1 / \Lambda^{2}\right)$ approximation.
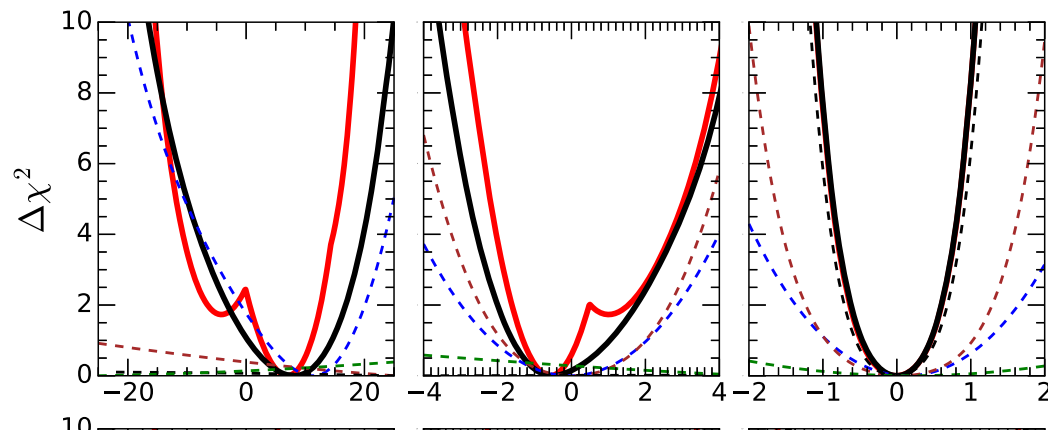

- 21 OP Global STXS $O\left(\Lambda^{-4}\right)$

- 12 OP EWPD+ EWDBD $O\left(\Lambda^{-4}\right)$

- 12 OP EWPD+ EWDBD WW $\mathcal{O}\left(\Lambda^{-4}\right)$

- 12 OP EWPD+ EWDBD WZ $O\left(\Lambda^{-4}\right)$

- 12 OP EWPD+ EWDBD W $\gamma \mathcal{O}\left(\Lambda^{-4}\right)$

- - 12 OP EWPD+ EWDBD Zjj $O\left(\Lambda^{-4}\right)$
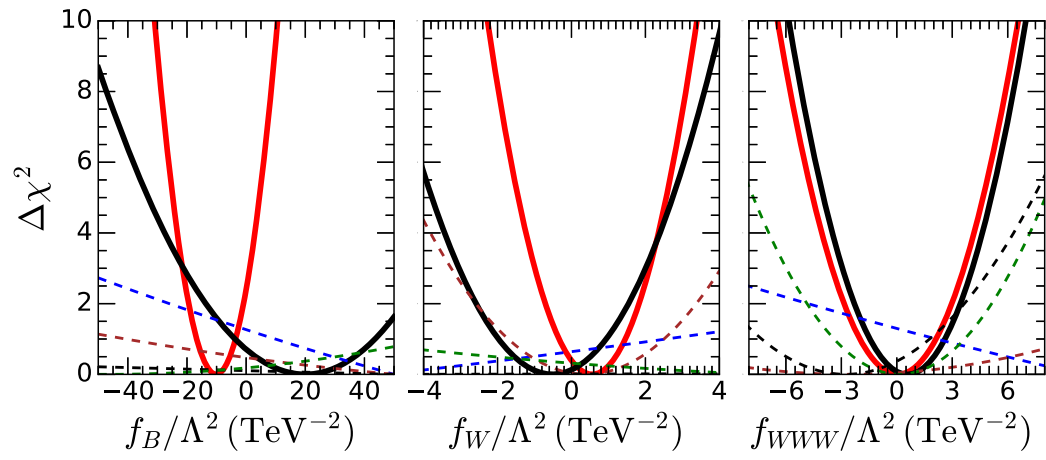

- 21 OP Global STXS $O\left(\Lambda^{-2}\right)$

- 12 OP EWPD+ EWDBD $O\left(\Lambda^{-2}\right)$

- 12 OP EWPD+ EWDBD WW $O\left(\Lambda^{-2}\right)$

- 12 OP EWPD+ EWDBD WZ $O\left(\Lambda^{-2}\right)$

- 12 OP EWPD+ EWDBD W $\gamma \mathcal{O}\left(\Lambda^{-2}\right)$

- 12 OP EWPD+ EWDBD Zjj $\mathcal{O}\left(\Lambda^{-2}\right)$

FIG. 3. $\Delta \chi^{2}$ dependence on the $f_{B} / \Lambda^{2}$ (left panels), $f_{W} / \Lambda^{2}$ (central panels), and $f_{W W W} / \Lambda^{2}$ (right panels) parameters after the marginalization over the 11 (20) undisplayed fit parameters for the analysis of EWDBD + EWPD (global STXS) data, as labeled in the figure. The upper panels show the results of our analysis using terms up to $\mathcal{O}\left(\Lambda^{-4}\right)$ in the Wilson coefficients, while the lower ones retained only the $\mathcal{O}\left(\Lambda^{-2}\right)$ terms. 

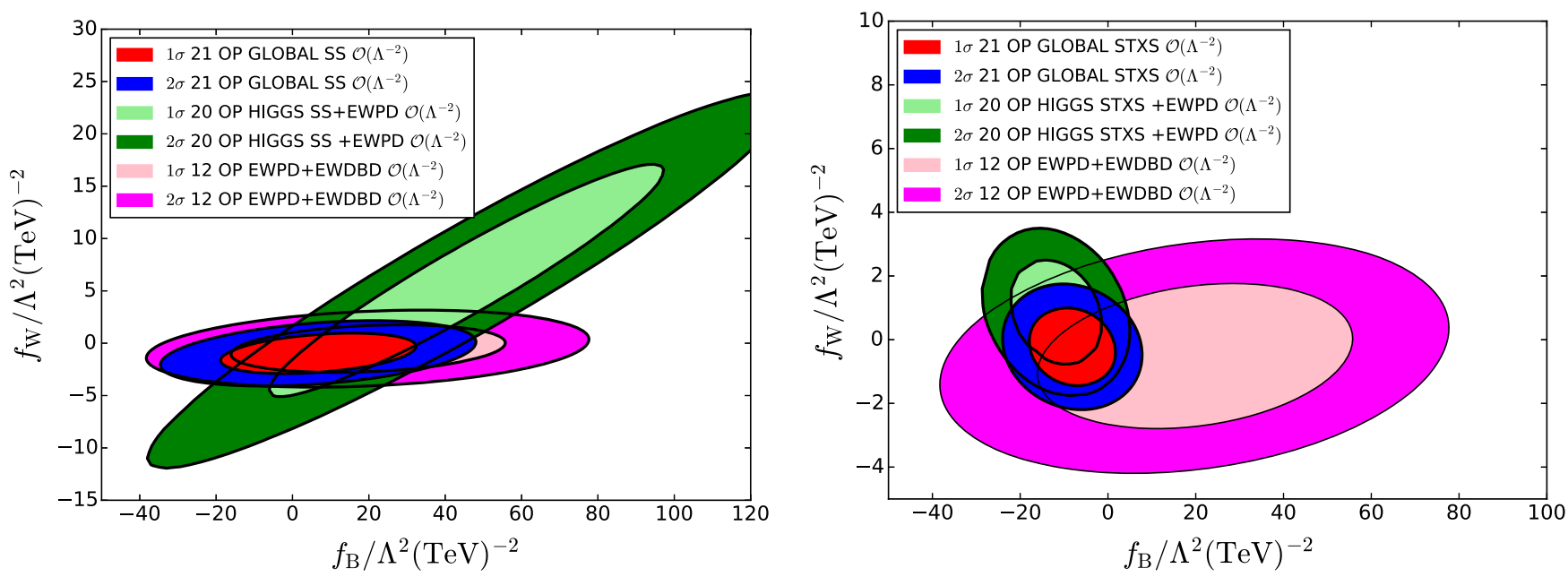

FIG. 4. $1 \sigma$ and $2 \sigma$ (2 d.o.f.) allowed regions on the $f_{B} / \Lambda^{2} \times f_{W} / \Lambda^{2}$ plane obtained from the analysis of different combinations of data samples as labeled in the figure. All results use predictions up to the $\mathcal{O}\left(\Lambda^{-2}\right)$ terms in the Wilson coefficients and have been marginalized over the undisplayed ones.
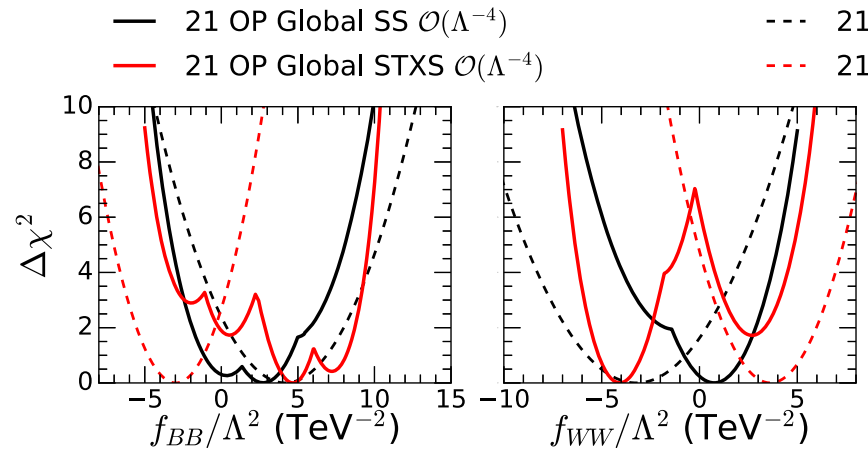

21 OP Global SS $\mathcal{O}\left(\Lambda^{-2}\right)$

- 21 OP Global STXS $\mathcal{O}\left(\Lambda^{-2}\right)$
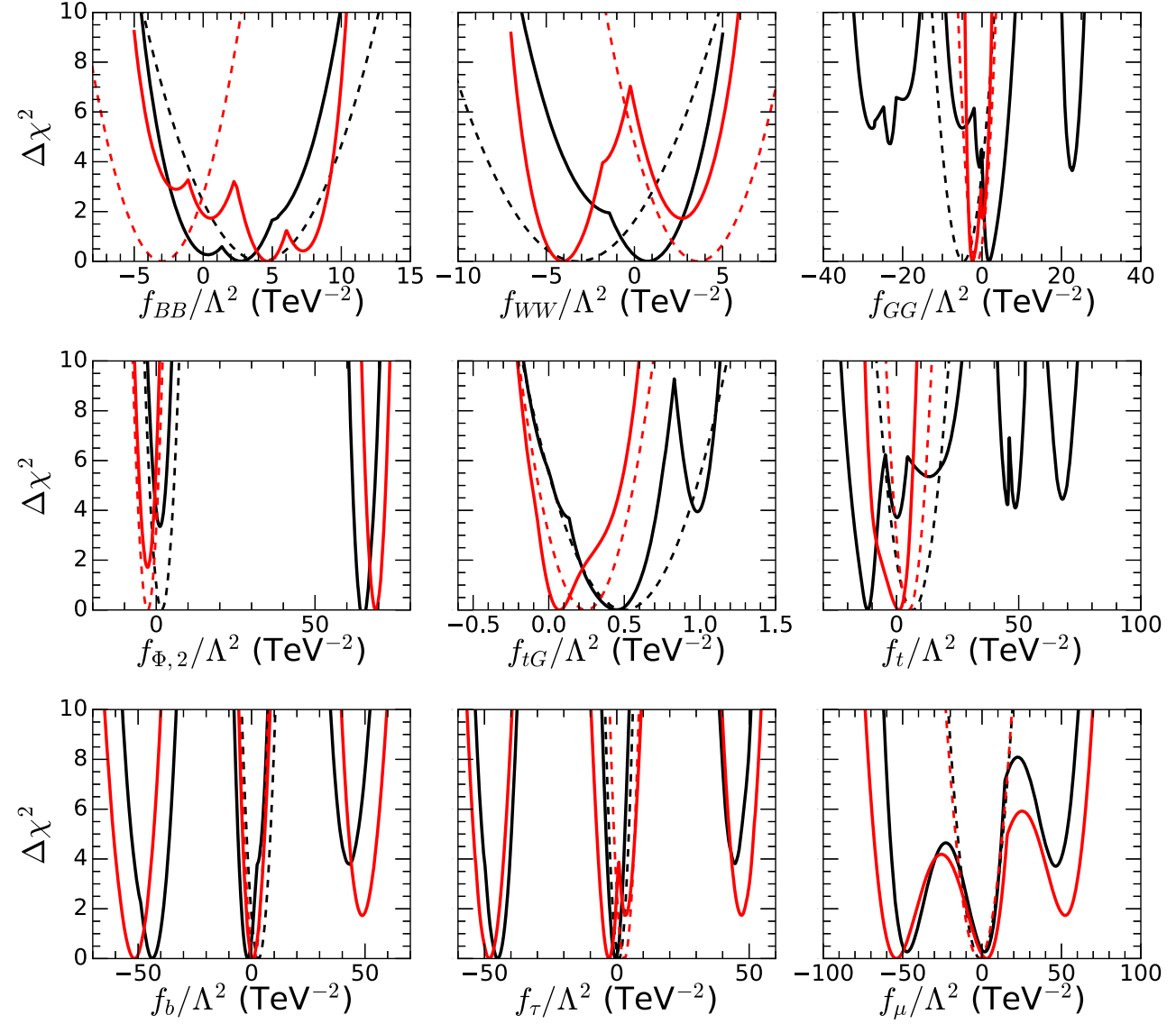

FIG. 5. Marginalized $\Delta \chi^{2}$ distributions as a function of the Wilson coefficients for the nine operators that only affect the Higgs physics and for the four variants of the global analysis as labeled in the figure (see text for details). 


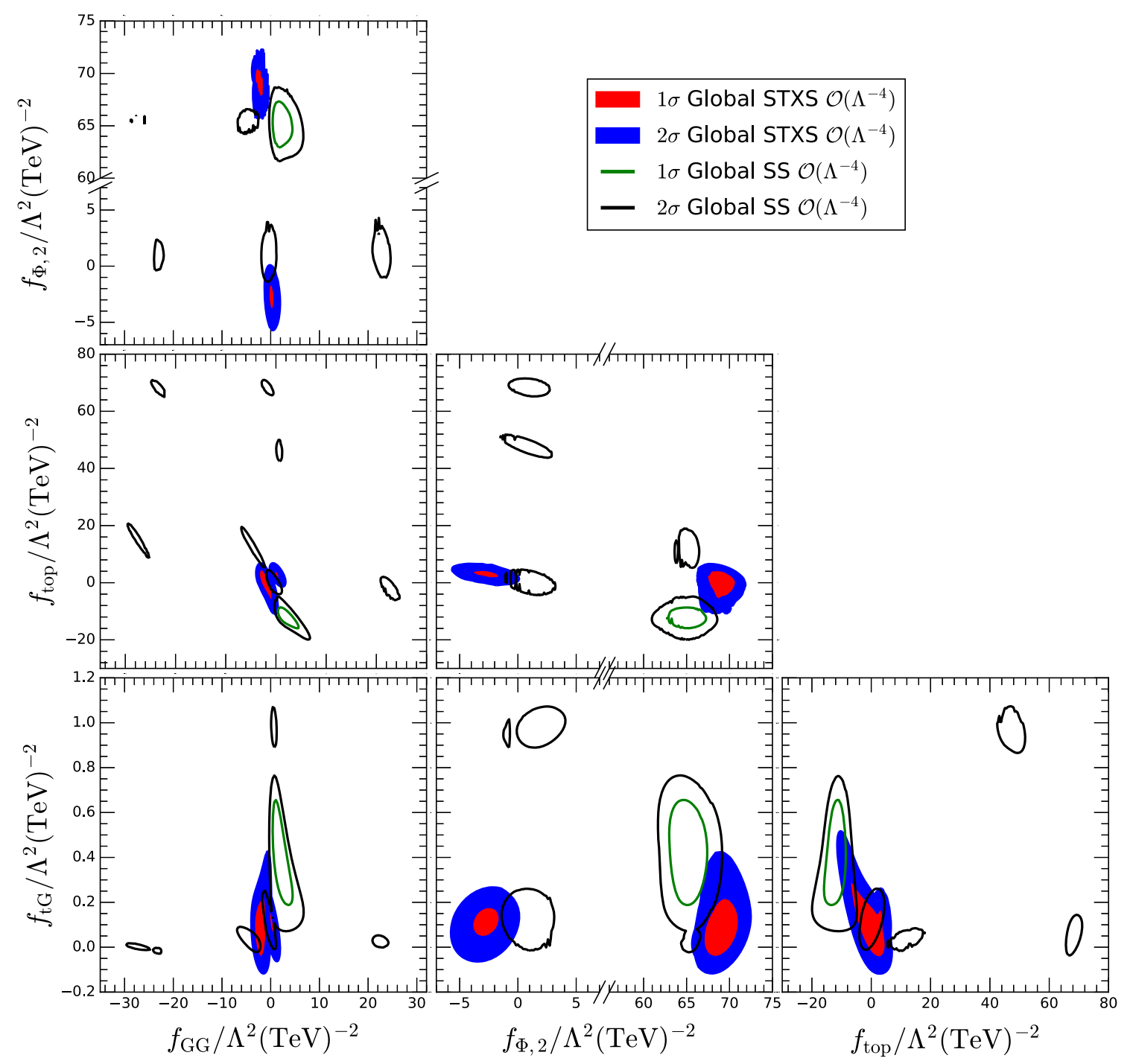

FIG. 6. $1 \sigma$ and 95\% C.L. (2 d.o.f.) allowed regions from the SS and STXS global analyses for the coefficients of the operators entering into the gluon-gluon Higgs vertex [Eq. (2.9)]. These results were obtained using terms up to $\mathcal{O}\left(\Lambda^{-4}\right)$ of the theoretical prediction, and the color code indicates the used dataset as labeled in the figure.

bounds imposed by the EWPD on the relevant quark operators with those from the global STXS analysis including up to $\mathcal{O}\left(\Lambda^{-2}\right)$ and up to $\mathcal{O}\left(\Lambda^{-4}\right)$ terms. Comparing these with the corresponding panels in Fig. 1, we see that the combination of EWPD with the LHC observables results in the quoted improvement on the bounds for those operators contributing to the light quark couplings. Conversely, $f_{\Phi, d_{33}}^{(1)}$ is only marginally affected by the inclusion of the EWDBD and Higgs data, and its best fit remains no-zero at $\sim 2 \sigma$ in the global analysis.

Finally, the lower panels of Fig. 1 display the marginalized $\Delta \chi^{2}$ for $f_{\Phi, e}^{(1)} / \Lambda^{2}$ and $f_{L L L L} / \Lambda^{2}$. The global analyses do not lead to significant improvement on the determination of these couplings. This is expected, since the operator $\mathcal{O}_{\Phi, e}^{(1)}$ modifies the $Z$ coupling to right-handed leptons, which were very precisely tested at LEP. On the contrary, it enters the LHC observables only via its contribution to the decay rate of the $Z$ boson to leptons in some of the final states considered. In other words, the dominant dependence of the global analysis on these coefficients still resides in the EWPD. The main effect of the inclusion of the LHC results is indirect via the restriction of the allowed range of variation of the other coefficients entering into the EWPD analysis.

\section{B. Triple anomalous gauge coupling constraints}

We present our results on the Wilson coefficients of the TGC operators $\mathcal{O}_{B}, \mathcal{O}_{W}$, and $\mathcal{O}_{W W W}$ in Fig. 3. We first consider the 12-dimensional parameter space 

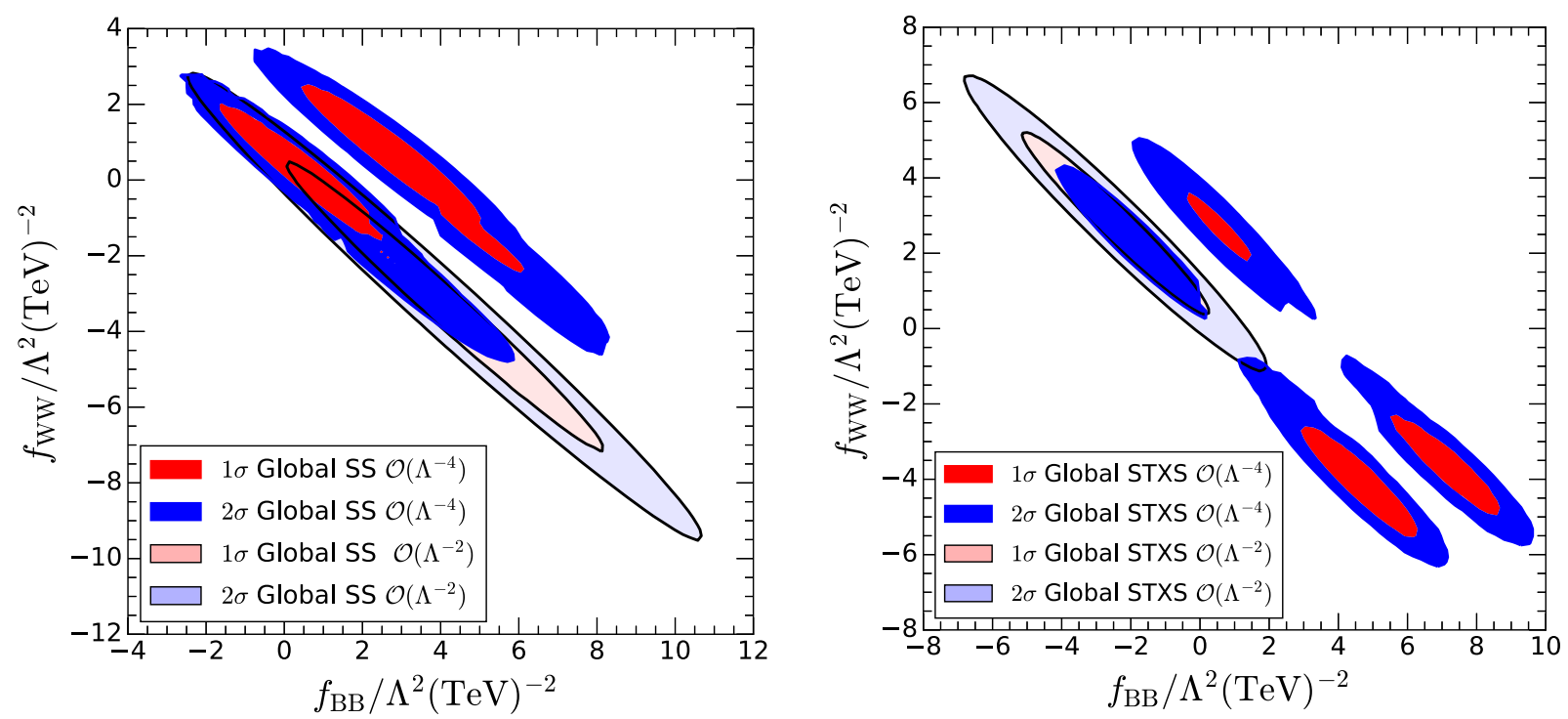

FIG. 7. $1 \sigma$ and $95 \%$ C.L. (2 d.o.f.) allowed regions from the (STXS) SS global analysis in the plane $f_{B B} / \Lambda^{2} \times f_{W W} / \Lambda^{2}$, shown in the left (right) panel. These results were obtained by using the theoretical predictions up to $\mathcal{O}\left(\Lambda^{-2}\right)$ or up to $\mathcal{O}\left(\Lambda^{-4}\right)$ approximations, as indicated by the color code.
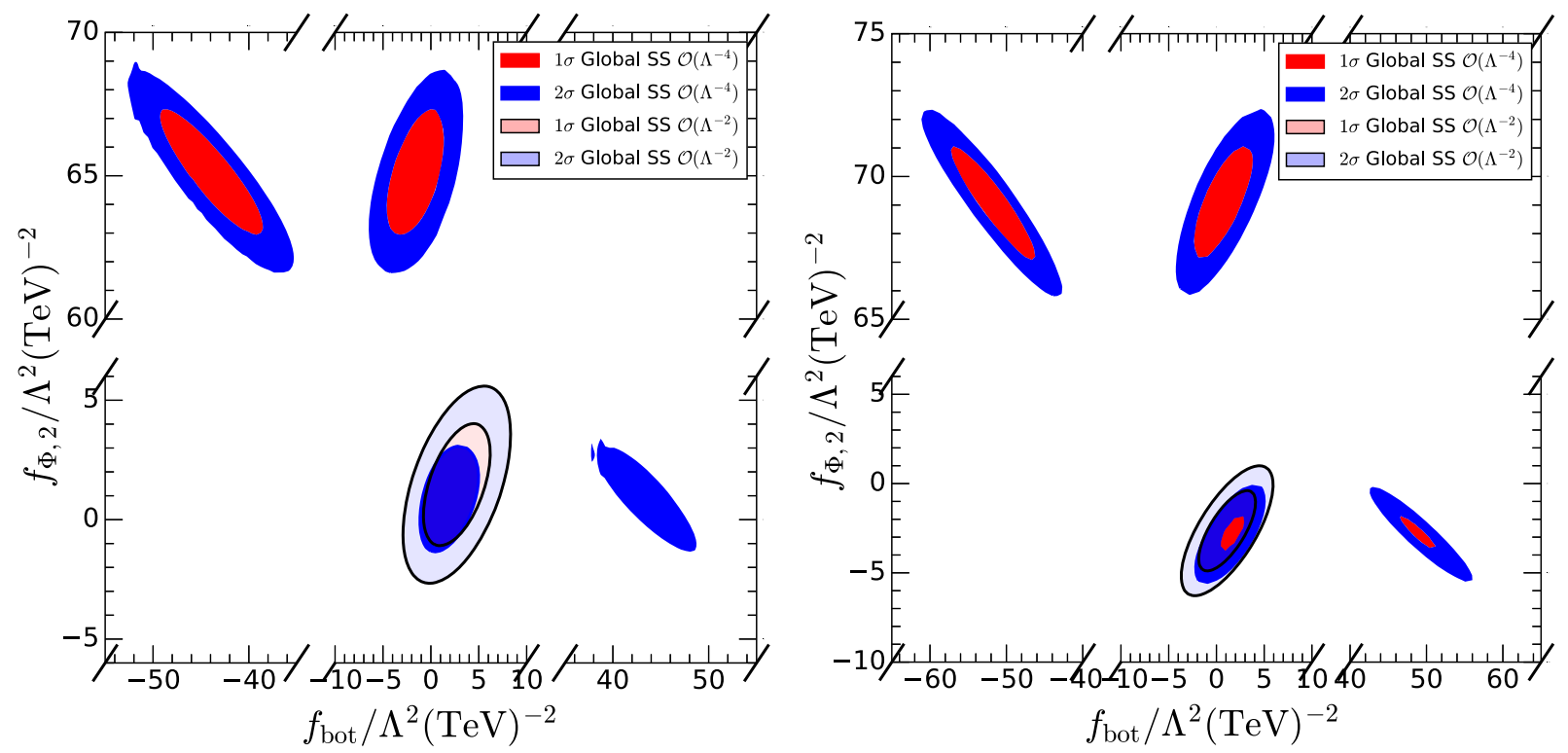

FIG. 8. $1 \sigma$ and 95\% C.L. (2 d.o.f.) allowed regions from the STXS global analysis in the plane $f_{\Phi, 2} / \Lambda^{2} \times f_{b} / \Lambda^{2}$. These results were obtained using the Higgs SS and STXS data, keeping the quadratic terms of the theoretical prediction, as indicated by the color code.

$$
\begin{aligned}
& \left\{f_{B}, f_{W}, f_{W W W}, f_{B W}, f_{\Phi, 1}, f_{\Phi, Q}^{(3)}, f_{\Phi, Q}^{(1)}, f_{\Phi, u}^{(1)}, f_{\Phi, d}^{(1)},\right. \\
& \left.\quad f_{\Phi, u d}^{(1)}, f_{\Phi, e}^{(1)}, f_{L L L L}\right\}
\end{aligned}
$$

and include the EWDBD and EWPD. The upper (lower) panels contain the one-dimensional $\Delta \chi^{2}$ distributions after marginalization over the 11 undisplayed coefficients, and they were obtained using the up-to-quadratic (up-to-linear) contributions of the 12 Wilson coefficients. Each panel shows as dashed lines the impact of the different diboson datasets, and as a full black line the combined EWPD + EWDBD analysis. For comparison, we also show the corresponding projections obtained from the global STXS fit performed in the 21-parameter space (red lines).

Respecting $f_{B} / \Lambda^{2}$, we find that EWPD + EWDBD provides only loose constraints on this parameter at $\mathcal{O}\left(\Lambda^{-2}\right)$ (see the black line in the lower-left panel of Fig. 3). Comparing this with the black line in the upperleft panel of the figure, we see how the inclusion of the $\mathcal{O}\left(\Lambda^{-4}\right)$ terms leads to better bounds, with the $W W$ channel 
(blue dashed line) playing the dominant role. This is so even though the integrated luminosity is larger for the $W \gamma$ data than for $W W$, because the $W \gamma$ data depend on the combination $\left(f_{B} / \Lambda^{2}+f_{W} / \Lambda^{2}\right)$, which allows for cancellations between $f_{B} / \Lambda^{2}$ and $f_{W} / \Lambda^{2}$. Similarly, the $W Z$ channel also depends on $f_{B} / \Lambda^{2}$ in another linear combination with $f_{W} / \Lambda^{2}$, and furthermore, its coefficient is suppressed by a factor $\tan ^{2} \theta_{W}$ with respect to $f_{W} / \Lambda^{2}$. From these panels we also conclude that $f_{B} / \Lambda^{2}$ is much more strongly constrained by the global analysis (red lines) than by EWPD + EWDBD. This is so because $\mathcal{O}_{B}$ contributes to both Higgs production and decay. This fact was observed before in Ref. [98]. But we find now that with the large run 2 luminosity and the STXS information, the constraints on $f_{B} / \Lambda^{2}$ are substantially improved even when including the dimension-six parameters only up to $\mathcal{O}\left(\Lambda^{-2}\right)$.

The $\Delta \chi^{2}$ distributions for $f_{W} / \Lambda^{2}$ are displayed in the middle panels, where we see that the dominant diboson channels constraining $f_{W} / \Lambda^{2}$ are the $W W$ and $W Z$ channels. Here too, $W \gamma$ production is not able to produce any sensible bound on $f_{W} / \Lambda^{2}$ due to its (anti)correlation with $f_{B} / \Lambda^{2}$, as previously commented. In this case, the limits obtained at $\mathcal{O}\left(\Lambda^{-2}\right)$ are already rather stringent, and they are significantly improved by the global analysis.

In order to show the importance of the Higgs data in constraining $f_{B} / \Lambda^{2}$ and $f_{W} / \Lambda^{2}$, we present in Fig. 4 the $1 \sigma$ and $2 \sigma$ allowed regions of the plane $f_{B} / \Lambda^{2} \times f_{W} / \Lambda^{2}$ obtained with different data samples. These results were obtained using only the contributions up to $\mathcal{O}\left(\Lambda^{-2}\right)$ on the Wilson coefficients and the SS (STXS) Higgs data in the left (right) panel. As we can see, the EWPD + EWDBD datasets lead to a very large allowed region (shown in pink). The constraints coming from the SS Higgs data (shown in the green regions on the left panel in combination with the EWPD) are also loose, but they are important to improve the limits when combined also with the EWDBD. But most interestingly, using only the Higgs STXS analysis in combination with EWPD (right panel), we are able to place much stronger bounds on $f_{W} / \Lambda^{2}$ and $f_{B} / \Lambda^{2}$; compare the green and pink regions. Clearly, the global fit on $\mathcal{O}_{B}$ is dominated by the data samples in the Higgs STXS analysis, while the constraints on $\mathcal{O}_{W}$ receive comparable contributions from Higgs STXS and EWDBD.

Finally, the right panels of Fig. 3 contain the dependence of the marginalized $\Delta \chi^{2}$ on $f_{W W W} / \Lambda^{2}$. In contrast to $f_{B} / \Lambda^{2}$ and $f_{W} / \Lambda^{2}$, the channel $W \gamma$ plays a significant role in constraining $f_{W W W} / \Lambda^{2}$ due to the use of kinematic distributions specially chosen to avoid the cancellation of the $1 / \Lambda^{2}$ contribution $[99,100]$. In fact, we can see from the lower-right panel of Fig. 3 that the most important contributions to the EWPD + EWDBD analysis originate from those channels ( $W \gamma$ and $Z j j$ ). Also, as expected, the global analysis has barely any additional impact on limiting
$\mathcal{O}_{W W W}$, since this operator does not contribute to the Higgs observables.

\section{Higgs couplings}

In order to probe for deviations from the SM predictions for the Higgs couplings, we have performed four global fits including the effects of the 21 operators in Eqs. (2.2)-(2.4) under different assumptions. As mentioned in Sec. III, in order to access the importance of the newly available kinematic distributions, we make two analyses: One in which that information is not included (Global SS) and another one in which it is (Global STXS). And, as for EWDBD, we make two variants of the analyses: one employing the theoretical predictions up to $\mathcal{O}\left(\Lambda^{-2}\right)$ terms in the Wilson coefficients, and one including up to $\mathcal{O}\left(\Lambda^{-4}\right)$ terms.

We have discussed in the context of Figs. 1 and 3 the results from these global analyses for the 12 operators that contribute also to the EWPD and EWDBD; therefore, we focus here on the nine operators not studied yet. We show in Fig. 5 the dependence of the marginalized $\Delta \chi_{\text {global }}^{2}$ on each of these nine Wilson coefficients for the four analysis variants.

From Fig. 5 we observe, as expected, that for the analysis including only the contributions up to $\mathcal{O}\left(\Lambda^{-2}\right)$ (dashed lines), there is a unique minimum in either the global SS or global STXS analysis. In comparing the red and black dashed lines, we conclude that at $\mathcal{O}\left(\Lambda^{-2}\right)$, the impact of the STXS observables in the overall picture amounts to an uncertainty reduction of $30 \%-40 \%$ for some of the bosonic operators, as well as a shift in the allowed region in a few cases.

Conversely, we also see in Fig. 5 that for the analysis including up to $\mathcal{O}\left(\Lambda^{-4}\right)$ terms and the SS samples for the Higgs data (solid black lines), all panels present some set of (quasi)degenerated minima. They are a direct reflection of the (quasi)degeneracies in the Higgs couplings in Eqs. (2.6)-(2.9). Comparing the solid black and red lines, we see the relevance of the kinematic distributions in resolving some of these degeneracies.

First, let us focus on the middle-left panel of Fig. 5, which depicts the $\Delta \chi^{2}$ distribution as a function of $f_{\Phi, 2} / \Lambda^{2}$. In the SS analysis at $\mathcal{O}\left(\Lambda^{-4}\right)$, there are two clearly almost degenerate minima associated with the flipping of sign of all Higgs couplings discussed below Eq. (2.6) for $f_{\Phi, 2} / \Lambda^{2} \simeq 65 \mathrm{TeV}^{-2}$. In fact, we find that the analysis shows a slight preference for this nonstandard solution. As seen in the figure, this is still the case once the information of the STXS observables is included but the $\Delta \chi^{2}$ of the SMconnected solution is reduced to $\sim 1.3 \sigma$.

The power of the kinematic distributions is particularly striking for the coefficients $f_{G G} / \Lambda^{2}, f_{t} / \Lambda^{2}$, and $f_{t G} / \Lambda^{2}$, which, together with $f_{\Phi, 2} / \Lambda^{2}$, enter into the effective gluon-gluon Higgs vertex, and for which the marginalized 
$\chi^{2}$ for the global SS analysis shown in the black curves in Fig. 5 presents a complex structure of local minima. This is further illustrated in Fig. 6. In this figure, we show the allowed regions from the global analysis performed at $\mathcal{O}\left(\Lambda^{-4}\right)$, projected over pairs of these parameters (after marginalization over the 19 undisplayed parameters in each panel). We observe the complex structure of allowed regions in $f_{G G} / \Lambda^{2} \times f_{\Phi, 2} / \Lambda^{2}, \quad f_{G G} / \Lambda^{2} \times f_{t} / \Lambda^{2}$, and $f_{t G} / \Lambda^{2} \times f_{\Phi, 2} / \Lambda^{2}$ that appear in the $\operatorname{SS} \mathcal{O}\left(\Lambda^{-4}\right)$ analysis. ${ }^{4}$ As inferred from the figure, the inclusion of the Higgs kinematic distributions in the analysis is able to fully separate the contributions from $\mathcal{O}_{G G}, \mathcal{O}_{t G}$, and $\mathcal{O}_{u \Phi, 33}$, while the degeneracy associated with $\mathcal{O}_{\Phi, 2}$ remains.

We now turn to the operators involved in the photonphoton-Higgs vertex. The upper-left and upper-central panels of Fig. 5 contain $\Delta \chi^{2}$ as functions of $f_{B B} / \Lambda^{2}$ and $f_{W W} / \Lambda^{2}$, respectively. The four analyses, SS and STXS with predictions at $\mathcal{O}\left(\Lambda^{-2}\right)$ and $\mathcal{O}\left(\Lambda^{-4}\right)$, lead to a unique allowed range for both Wilson couplings compatible with the SM at the $2 \sigma$ level, but for the STXS analysis at $\mathcal{O}\left(\Lambda^{-4}\right)$ we still observe several minima. To better understand these results, we present in Fig. 7 the $1 \sigma$ and 95\% C.L. twodegree-of-freedom ( 2 d.o.f.) allowed regions from the SS and STXS global analyses in the plane $f_{B B} / \Lambda^{2} \times f_{W W} / \Lambda^{2}$. From the figures we see that all allowed regions display the strong anticorrelation between those parameters associated with the $\left(f_{W W}+f_{B B}\right) / \Lambda^{2}$ dependence of the Higgsphoton-photon vertex. This correlation is not exact, because it is broken by other measurements - in particular, by the $H F_{\mu \nu} Z^{\mu \nu}$ branching ratio, which constrains a different combination: $f_{W W} / \Lambda^{2}$ and $f_{B B} / \Lambda^{2}$. In the figure, we also observe the existence of the second allowed region(s) around $\left(f_{W W}+f_{B B}\right) / \Lambda^{2} \sim 3 \mathrm{TeV}^{-2}$ in the analysis performed to $\mathcal{O}\left(\Lambda^{-4}\right)$, associated with the flipping of sign of the Higgs-photon-photon coupling with respect to the SM value discussed below Eq. (2.8). In this case, unlike for the gluon-gluon-Higgs vertex, the kinematic information contained in the STXS observables cannot resolve these two solutions, for which the kinematics is identical. In addition, there are two solutions for each of the mirror solutions associated with the degeneracy associated with $f_{\Phi, 2}$, which affects the production cross section. For the STXS analysis, the two solutions become separated enough to lead to the two additional disconnected regions observed in the right panel. Further details can be obtained from the correlation matrices presented in the Appendix.

In respect to the Yukawa couplings $H f f$, as discussed in Sec. II, they exhibit a fourfold degeneracy in the $\mathcal{O}\left(\Lambda^{-4}\right)$ analysis because the sign of this coupling can be flipped by $f_{\Phi, 2} / \Lambda^{2}$ and $f_{f} / \Lambda^{2}$; see Eq. (2.7). This degeneracy favors

\footnotetext{
${ }^{4}$ We notice that the fact that the external bias on $f_{t G}$ from top observables [Eq. (3.4)] is not centered at zero further adds to the complex structure of local minima.
}

the existence of three disconnected allowed ranges [33], and it is clearly observable in both analyses at $\mathcal{O}\left(\Lambda^{-4}\right)$ in the three lower panels in Fig. 5 for the bottom, $\tau$, and $\mu$ Yukawas, as well as in the left panel of Fig. 8. These solutions are not totally degenerate because, as discussed above, in the $\mathcal{O}\left(\Lambda^{-4}\right)$ analysis we find a slight preference for the nonstandard solution for $f_{\Phi, 2} / \Lambda^{2}$. Including the kinematic information in the form of the STXS observables does not resolve this degeneracy.

For $f_{t} / \Lambda^{2}$, the fourfold degeneracy is expected to be partially broken, since the scattering amplitude for the $t H$ production receives contributions from the $t t H$ and $V V H$ vertices, being sensitive to the relative signs of the different diagrams contributing [101-103]. Conversely, the contribution of this coupling to the effective gluon-gluon-Higgs vertex introduces the additional degeneracy and correlations with $f_{G G}$ and $f_{t G}$ described above. Altogether, we find that the STXS Higgs data is able to constrain univocally the top Yukawa coupling even at $\mathcal{O}\left(\Lambda^{-4}\right)$.

We present the $1 \sigma$ bounds and correlations in the Appendix for the $\mathcal{O}\left(\Lambda^{-2}\right)$ global SS and STXS analyses. From these results, we can see that the strongest (anti) correlations are between the pairs of operators $\mathcal{O}_{B} \otimes \mathcal{O}_{B B}$, $\mathcal{O}_{B} \otimes \mathcal{O}_{W W}, \mathcal{O}_{B B} \otimes \mathcal{O}_{W W}, \mathcal{O}_{G G} \otimes \mathcal{O}_{u \Phi, 33}, \mathcal{O}_{G G} \otimes \mathcal{O}_{t G}$, $\mathcal{O}_{B W} \otimes \mathcal{O}_{\Phi, 1}$, and $\mathcal{O}_{\Phi, e}^{(1)} \otimes \mathcal{O}_{L L L L}$. The first three stem mainly from the Higgs decay into photon pairs, while the next two are due to the Higgs coupling to gluons, and the last two are dominantly due to their contribution to the EWPD observables. Furthermore, $\mathcal{O}_{\Phi, 2}$ possesses sizable correlations with many dimension-six operators due to the possibility of flipping the sign of the Higgs couplings.

\section{RESULTS FOR SIMPLIFIED MODELS}

Figures 9 and 10 contain the results of the analyses for the simplified models presented in Sec. II A.

The $\Delta \chi^{2}$ distribution for the singlet scalar extension of the SM is presented in the left panel of Fig. 9, for both global analysis and the predictions obtained up to $\mathcal{O}\left(\Lambda^{-2}\right)$ and up to $\mathcal{O}\left(\Lambda^{-4}\right)$, which lead to similar results. We find, for example, that considering only the effects at order $\mathcal{O}\left(\Lambda^{-2}\right)$, the bound obtained from the SS analysis is

$$
|\sin \theta|<0.279
$$

at a $95 \%$ C.L. This model only generates $f_{\Phi, 2} / \Lambda^{2}$ at tree level; therefore, we can translate the above limit into $f_{\Phi, 2} / \Lambda^{2}<1.4 \mathrm{TeV}^{-2}$ using Eq. (2.10). Notice that this constraint is about 4 times stronger than the limit originating from the 21-parameter analysis; see Table VI. Also, this limit is similar to that derived in Ref. [80].

The $\Delta \chi^{2}$ distributions for the simplified models that contain the addition of one state as described in Table III can be found in the right panel of Fig. 9. For concreteness, we show the results for the global STXS analysis at 

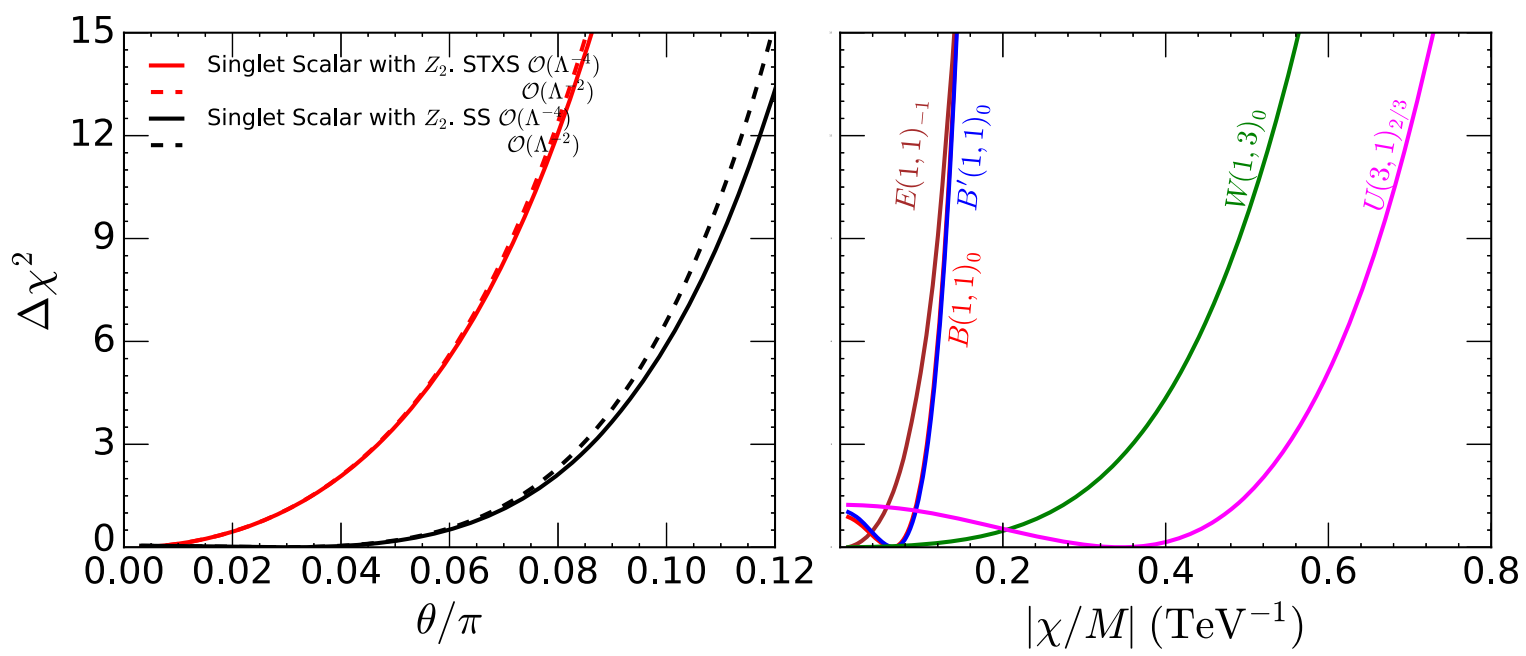

FIG. 9. The left (right) panel contains $\Delta \chi^{2}$ as a function of $\theta / \pi(\chi / M)$ for the singlet scalar model (models with one additional state). The results shown in the right panel correspond to the global analysis with the STXS Higgs datasets and including only the contributions that are linear on the Wilson coefficients $\left[\mathcal{O}\left(\Lambda^{-2}\right)\right]$.
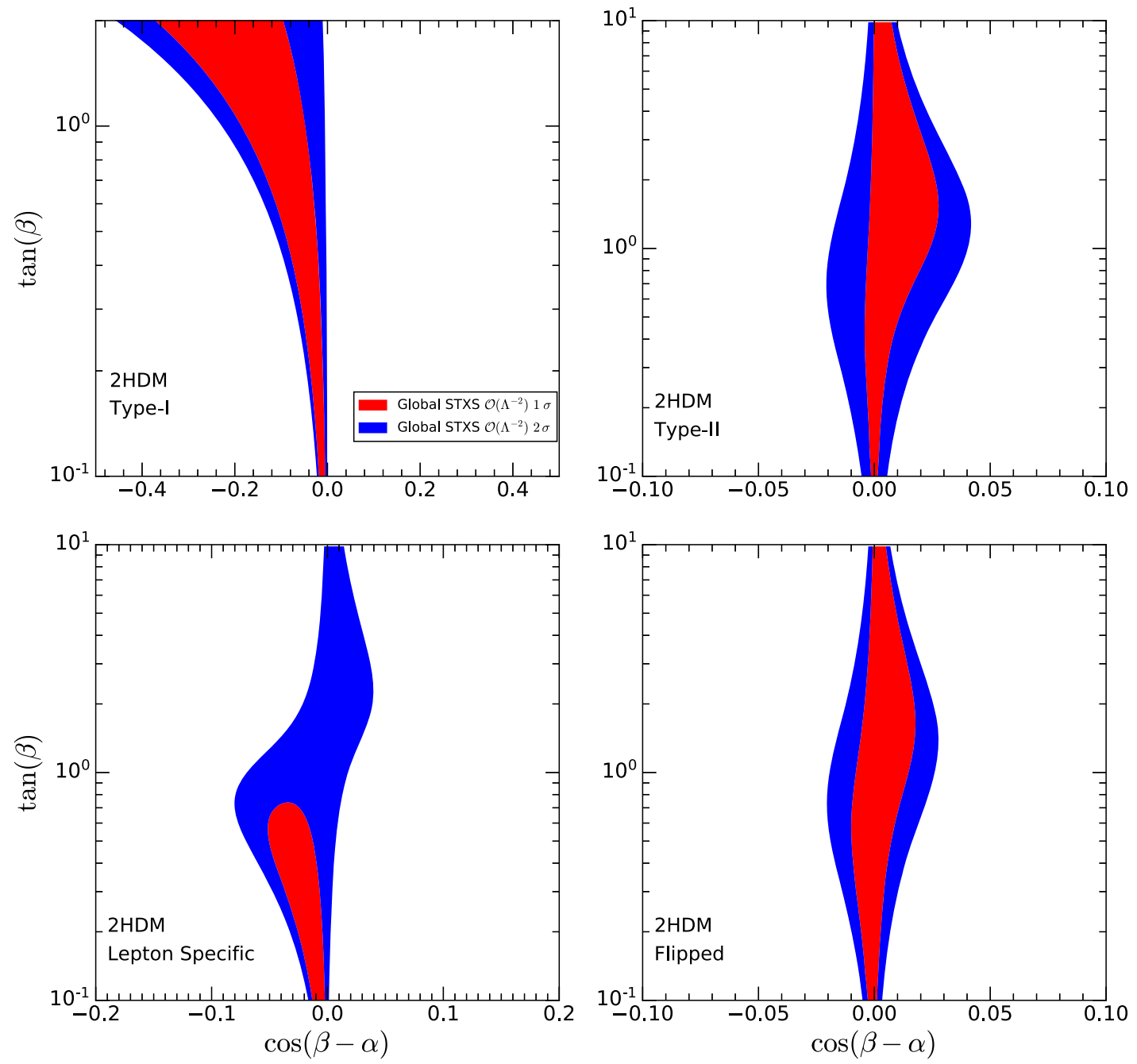

FIG. 10. Allowed regions of the plane $\cos (\beta-\alpha) \times \tan \beta$ for several two-Higgs-doublet models. The results shown correspond to the global analyses with the STXS Higgs datasets, including only the contributions that are linear on the Wilson coefficients $\left[\mathcal{O}\left(1 / \Lambda^{2}\right)\right]$. 
TABLE VI. Marginalized 95\% C.L. allowed ranges for the Wilson coefficients for the four different global analyses performed in this work.

\begin{tabular}{|c|c|c|c|c|}
\hline \multirow[b]{2}{*}{ Operator } & \multicolumn{4}{|c|}{$95 \%$ C.L. $f / \Lambda^{2}\left(\mathrm{TeV}^{-2}\right)$} \\
\hline & Global SS $\mathcal{O}\left(\Lambda^{-4}\right)$ & Global SS $\mathcal{O}\left(\Lambda^{-2}\right)$ & Global STXS $\mathcal{O}\left(\Lambda^{-4}\right)$, & Global STXS $\mathcal{O}\left(\Lambda^{-2}\right)$, \\
\hline $\mathcal{O}_{B}$ & $(-9.8,14)$ & $(-5.5,37)$ & $(-11,15)$ & $(-23,3.0)$ \\
\hline $\mathcal{O}_{W}$ & $(-2.0,2.8)$ & $(-3.0,2.6)$ & $(-2.0,2.7)$ & $(-1.2,2.3)$ \\
\hline $\mathcal{O}_{W W W}^{\prime \prime}$ & $(-0.80,0.81)$ & $(-3.5,4.5)$ & $(-0.81,0.78)$ & $(-4.1,4.2)$ \\
\hline $\mathcal{O}_{B B}$ & $(-2.8,7.5)$ & $(-1.2,9.6)$ & $(-3.4,9.4)$ & $(-6.6,0.65)$ \\
\hline $\mathcal{O}_{W W}$ & $(-3.9,3.7)$ & $(-8.3,1.8)$ & $(-6.1,-1.7) \cup(0.78,4.5)$ & $(0.30,7.9)$ \\
\hline $\mathcal{O}_{G G}$ & $(-1.0,5.7) \cup(22,23)$ & $(-9.7,0.23)$ & $(-3.7,1.4)$ & $(-4.3,1.7)$ \\
\hline $\mathcal{O}_{t G}$ & $(0.11,0.71)$ & $(0.073,0.93)$ & $(-0.010,0.48)$ & $(-0.035,-0.53)$ \\
\hline $\mathcal{O}_{\Phi, 2}$ & $(0.33,2.0) \cup(62,68)$ & $(-1.7,5.2)$ & $(-4.7,-0.71) \cup(66,72)$ & $(-5.7,0.26)$ \\
\hline $\mathcal{O}_{u \Phi, 33}$ & $(-18,-7.3) \cup(-1.3,1.7)$ & $(-2.8,16)$ & $(-10,5.9)$ & $(-0.89,11)$ \\
\hline $\mathcal{O}_{d \Phi, 33}$ & $(-52,-37) \cup(-5.6,3.3) \cup(41,45)$ & $(-1.6,7.8)$ & $(-60,-44) \cup(-3.5,5.2) \cup(44,54)$ & $(-2.8,5.0)$ \\
\hline $\mathcal{O}_{e \Phi, 33}$ & $(-50,-40) \cup(-3.7,2.7) \cup(44,45)$ & $(-2.5,4.2)$ & $(-53,-43) \cup(-7.0,6.2) \cup(43,51)$ & $(-0.64,6.3)$ \\
\hline $\mathcal{O}_{e \Phi, 22}$ & $(-57,-28) \cup(-18,11) \cup(41,51)$ & $(-14,12)$ & $(-69,-30) \cup(-22,15) \cup(39,62)$ & $(-15,11)$ \\
\hline $\mathcal{O}_{B W}$ & $(-0.21,1.7)$ & $(-0.064,1.8)$ & $(-0.19,1.6)$ & $(-0.22,1.7)$ \\
\hline $\mathcal{O}_{\Phi, 1}$ & $(-0.040,0.14)$ & $(-0.024,0.16)$ & $(-0.037,0.14)$ & $(-0.037,0.14)$ \\
\hline $\mathcal{O}_{\Phi, Q}^{(3)}$ & $(-0.23,0.23)$ & $(-0.30,0.24)$ & $(-0.25,0.26)$ & $(-0.15,0.27)$ \\
\hline $\mathcal{O}_{\Phi, Q}^{(1)}$ & $(-0.041,0.10)$ & $(-0.091,0.085)$ & $(-0.034,0.11)$ & $(-0.098,0.075)$ \\
\hline $\mathcal{O}_{\Phi, u}^{(1)}$ & $(-0.22,0.24)$ & $(-0.34,0.22)$ & $(-0.26,0.29)$ & $(-0.41,0.094)$ \\
\hline $\mathcal{O}_{\Phi, d}^{(1)}$ & $(-0.42,0.10)$ & $(-0.95,0.0096)$ & $(-0.34,0.11)$ & $(-0.81,-0.054)$ \\
\hline $\mathcal{O}_{\Phi, u d}^{(1)}$ & $(-0.13,0.13)$ & $\ldots$ & $(-0.12,0.12)$ & $\cdots$ \\
\hline $\mathcal{O}_{\Phi, e}^{(1)}$ & $(-0.076,0.0040)$ & $(-0.081,-0.0016)$ & $(-0.072,0.0020)$ & $(-0.074,-0.0040)$ \\
\hline $\mathcal{O}_{L L L L}$ & $(-0.046,0.0035)$ & $(-0.047,0.0029)$ & $(-0.045,0.0046)$ & $(-0.046,0.0034)$ \\
\hline
\end{tabular}

$\mathcal{O}\left(\Lambda^{-2}\right)$, but for these models the inclusion of the quadratic terms has very little impact, indicating the stability of the results with respect to higher-order corrections, as well as the validity of the high-mass expansion. From the figure, we read the following $95 \%$ C.L. bounds on $\chi^{2} / M$, which imply the quoted mass limit for $\chi=1$ :

$$
\begin{array}{lll}
\frac{\chi}{M}<0.084 \mathrm{TeV}^{-1} & (M>12 \mathrm{TeV}) & \text { Model with } E(1,1)_{-1}, \\
\frac{\chi}{M}<0.15 \mathrm{TeV}^{-1} & (M>6.7 \mathrm{TeV}) & \text { Model with } B^{\prime}(1,1)_{0}, \\
\frac{\chi}{M}<0.16 \mathrm{TeV}^{-1} & (M>6.2 \mathrm{TeV}) & \text { Model with } B(1,1)_{-1}, \\
\frac{\chi}{M}<0.35 \mathrm{TeV}^{-1} & (M>2.8 \mathrm{TeV}) & \text { Model with } W(1,3)_{0}, \\
\frac{\chi}{M}<0.58 \mathrm{TeV}^{-1} & (M>1.7 \mathrm{TeV}) & \text { Model with } U(3,1)_{2 / 3} .
\end{array}
$$

The tightest constraint is for the model containing a new lepton $E$ (brown curve), since this model generates the strongly bound Wilson coefficients $f_{\Phi, e}^{(1)} / \Lambda^{2}$ and $f_{\Phi, 1} / \Lambda^{2}$, which appear when the Wilson coefficients generated at the high scale matching are rotated to the HISZ basis using Eqs. (2.12) and (2.13). The models with an extra vector $B$ are also subject to strong limits because they generate $f_{\Phi, 1} / \Lambda^{2}$-i.e., they contribute to the $T$ parameter.

The additional vector triplet $W$ and the extra vector quark $U$ prompt the appearance of the Wilson coefficient $f_{\Phi, Q}^{(3)} / \Lambda^{2}$, which is well constrained by the precise determination of the electroweak gauge couplings of left-handed quarks with EWPD and LHC diboson [37] and Higgs associated production [104,105] data. The extra vector quark $U$ also generates $f_{\Phi, Q}^{(1)} / \Lambda^{2}$ but with the opposite sign. $f_{\Phi, Q}^{(1)} / \Lambda^{2}$ also contributes to the couplings of the lefthanded quarks to the $Z$ boson, which leads to a small anticorrelation between these two coefficients. This results in the slighter weaker bounds in this model.

Figure 10 contains the constraints on $2 \mathrm{HDM}$ s that we obtain by performing the global analysis at $\mathcal{O}\left(\Lambda^{-2}\right)$ and using the STXS Higgs datasets. In the figure, we see that the allowed range for $\cos (\beta-\alpha)$ is tightly constrained in agreement with the alignment assumption, with the only exception being the Type-I model in the large $\tan \beta$ limit, for which the Wilson coefficients we are considering approach zero, and therefore no bound can be imposed in this approximation. In fact, with that exception, our results show that the allowed parameter space at the 95\% C.L. is strongly bounded. Compared to the experimental results obtained in Ref. [106] that used just a 
fraction of the run 2 integrated luminosity, ${ }^{5}$ we find stronger bounds on $\cos (\beta-\alpha)$ for fixed $\tan \beta$ by up to a factor of four, depending on the model and the sign of $\cos (\beta-\alpha)$. Also, in our analysis, we do not find the small lobe features observed in Ref. [106] for positive $\cos (\beta-\alpha)$ and large $\tan \beta$. Our bounds on Type-I and Type-II models are comparable with those derived in Refs. $[9,107]$.

\section{DISCUSSION}

In this work, we have presented the results of comprehensive analyses of low-energy electroweak precision measurements as well as LHC data on gauge boson pair production and Higgs observables in the context of the SMEFT. We focused on observables related to the electroweak sector, which at present allow for precision tests of the couplings between electroweak gauge bosons and fermions, triple electroweak gauge couplings, and the couplings of the Higgs to fermions and gauge bosons. For the sake of assessing the impact of the Higgs kinematic distributions, we performed an analysis with and without the STXS Higgs data in combination with the Higgs total signal strengths. In total, the global analyses of EWPD and EWDBD and Higgs results from LHC encompass 167 observables when considering only SS data and 255 observables when including the STXS samples; see Sec. III for further details.

We worked in the framework of effective Lagrangians, assuming the linear realization of the electroweak gauge symmetry. Dimension-six operators are those with the lowest dimension which contribute significantly to the considered processes at lowest order. The global analysis involves the 21 operators in Eq. (2.5) under the flavor assumption that the new operators do not introduce additional tree-level sources of flavor violation or violation of the universality of the weak current. Furthermore, we also analyzed the constraints on a few simplified models to illustrate how relations between the generated Wilson coefficients within specific models lead to tighter limits.

All of the analyses performed show no statistically significant source of tension with the SM. We find

$$
\begin{aligned}
\chi_{\min \text { EWPD+EWDBD }, \mathrm{SM}}^{2} & =91, & & 111 \text { observables }, \\
\chi_{\min \text { Global SS }, \mathrm{SM}}^{2} & =133, & & 166 \text { observables }, \\
\chi_{\min \text { Global STXS,SM }}^{2} & =304, & & 255 \text { observables },
\end{aligned}
$$

to be compared with

$$
\begin{array}{rlrl}
\chi_{\text {min } \operatorname{EWPD}+\operatorname{EWDBD}, \operatorname{SMEFT} \mathcal{O}\left(\Lambda^{-4}\right)\left[\operatorname{SMEFT} \mathcal{O}\left(\Lambda^{-2}\right)\right]}^{2} & =87[85], & 111 \text { observables \& } 12 \text { operators }, \\
\chi_{\min \operatorname{Global} \operatorname{SS}, \operatorname{SMEFT} \mathcal{O}\left(\Lambda^{-4}\right)\left[\operatorname{SMEFT} \mathcal{O}\left(\Lambda^{-2}\right)\right]}^{2} & =115[112], & 166 \text { observables } \& 21 \text { operators }, \\
\chi_{\text {min Global STXS,SMEFT } \mathcal{O}\left(\Lambda^{-4}\right)\left[\operatorname{SMEFT} \mathcal{O}\left(\Lambda^{-2}\right)\right]}^{2} & =266[264], & & 255 \text { observables } \& 21 \text { operators. }
\end{array}
$$

We summarize our results of the $\chi^{2}$ dependence on the Wilson coefficients for the $\mathcal{O}\left(\Lambda^{-2}\right)$ and $\mathcal{O}\left(\Lambda^{-4}\right)$ analyses, which we performed with the most comprehensive dataset including the kinematic information on the Higgs observables, by displaying the corresponding one-dimensional $\Delta \chi^{2}$ distributions shown in Fig. 11, where we marginalized over the 20 undisplayed variables in each panel. With these results and the corresponding ones for the global SS analysis, we obtain the $95 \%$ C.L. allowed ranges of the 21 Wilson coefficients that we present in Table VI, and we graphically display them in Fig. 12. The maximum allowed value for each Wilson coefficient at a given C.L. can be translated into a lower bound on an effective new physics scale

$$
\Lambda_{\min , \text { C.L. }} \equiv \frac{1}{\sqrt{\left|f / \Lambda^{2}\right|_{\max , \text { C.L. }}}},
$$

\footnotetext{
${ }^{5}$ Notice also that Ref. [106] obtained their constraints by adapting the so-called $\kappa$ framework, which contains quadratic terms in the anomalous couplings.
}

which is depicted in Fig. 13. Notice that $\Lambda_{\text {min,C.L. }}$ only coincides with the minimum energy scale in the operator expansion, $\Lambda$, for coefficients $f=1$. $\Lambda$ could be smaller than $\Lambda_{\min , \text { C.L. }}$ if the coupling is weak.

In brief, the main results that we would like to stress are the following:

(1) In our basis, there is no blind direction in the electroweak precision observables. Therefore, the limits originating from EWPD are already stringent on eight Wilson coefficients. In fact, the EWPD dominates the bounds on $\mathcal{O}_{L L L L}, \mathcal{O}_{\Phi, e}^{(1)}, \mathcal{O}_{\Phi, 1}, \mathcal{O}_{B W}$, and $f_{\Phi, Q}^{(1)} / \Lambda^{2}$ up to some small but not negligible contribution from LHC data (see Fig. 1).

(2) Conversely, the global analysis results in a sizable reduction of the uncertainty on $f_{\Phi, Q}^{(3)} / \Lambda^{2}$ with respect to the limits obtained using only EWPD. This is expected due to the role played by this operator in the Higgs associated production [104,105].

(3) The analysis of the EWPD of $f_{\Phi d}^{(1)} / \Lambda^{2}$ points towards a nonzero value for this coefficient due to the $2.7 \sigma$ discrepancy between the observed $A_{\mathrm{FB}}^{0, b}$ and the SM. Under the assumption that the operators modifying 

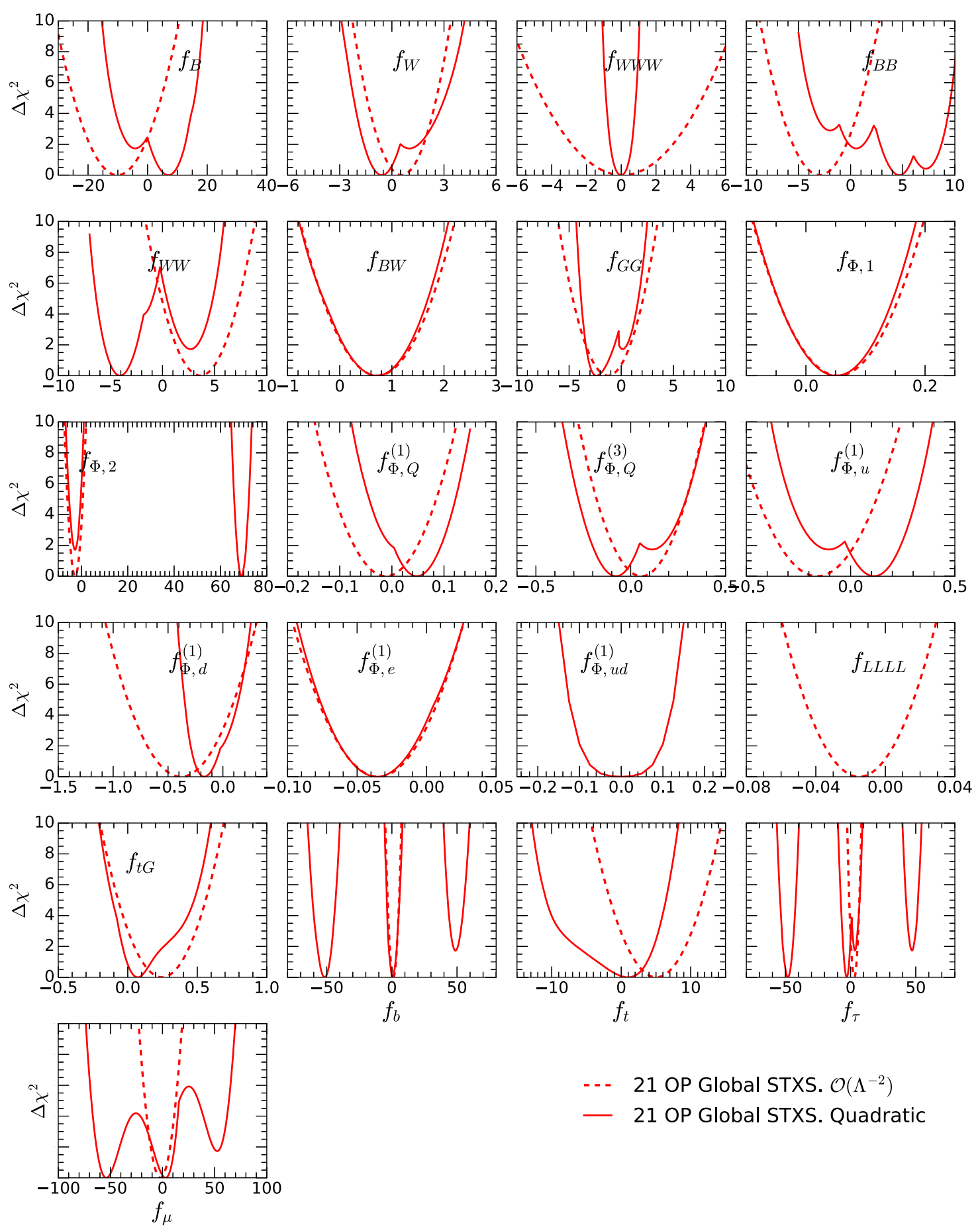

— 21 OP Global STXS. Quadratic

FIG. 11. Marginalized one-dimensional $\Delta \chi^{2}$ distributions for the 21 parameters appearing in our global fit including the STXS Higgs datasets. The dashed (solid) line stands for the results obtained with the theoretical predictions for the observables expanded at $\mathcal{O}\left(\Lambda^{-2}\right)$ $\left[\mathcal{O}\left(\Lambda^{-4}\right)\right]$ in the Wilson coefficients.

the fermion-gauge couplings are generation independent, the inclusion of the EWDBD and the Higgs data in the analysis (either SS or STXS) gives rise to a result compatible with the SM at better than 95\% C.L. and with slightly reduced errors. In addition, the global constraint on $f_{\Phi u}^{(1)} / \Lambda^{2}$ is also slightly improved by combining EWPD with the LHC results.

(4) Allowing for $f_{\Phi d}^{(1)} / \Lambda^{2}$ to be different for the bottom quark, the combination of EWPD with the LHC observables results in the quoted improvement on the bounds for those operators contributing to the 


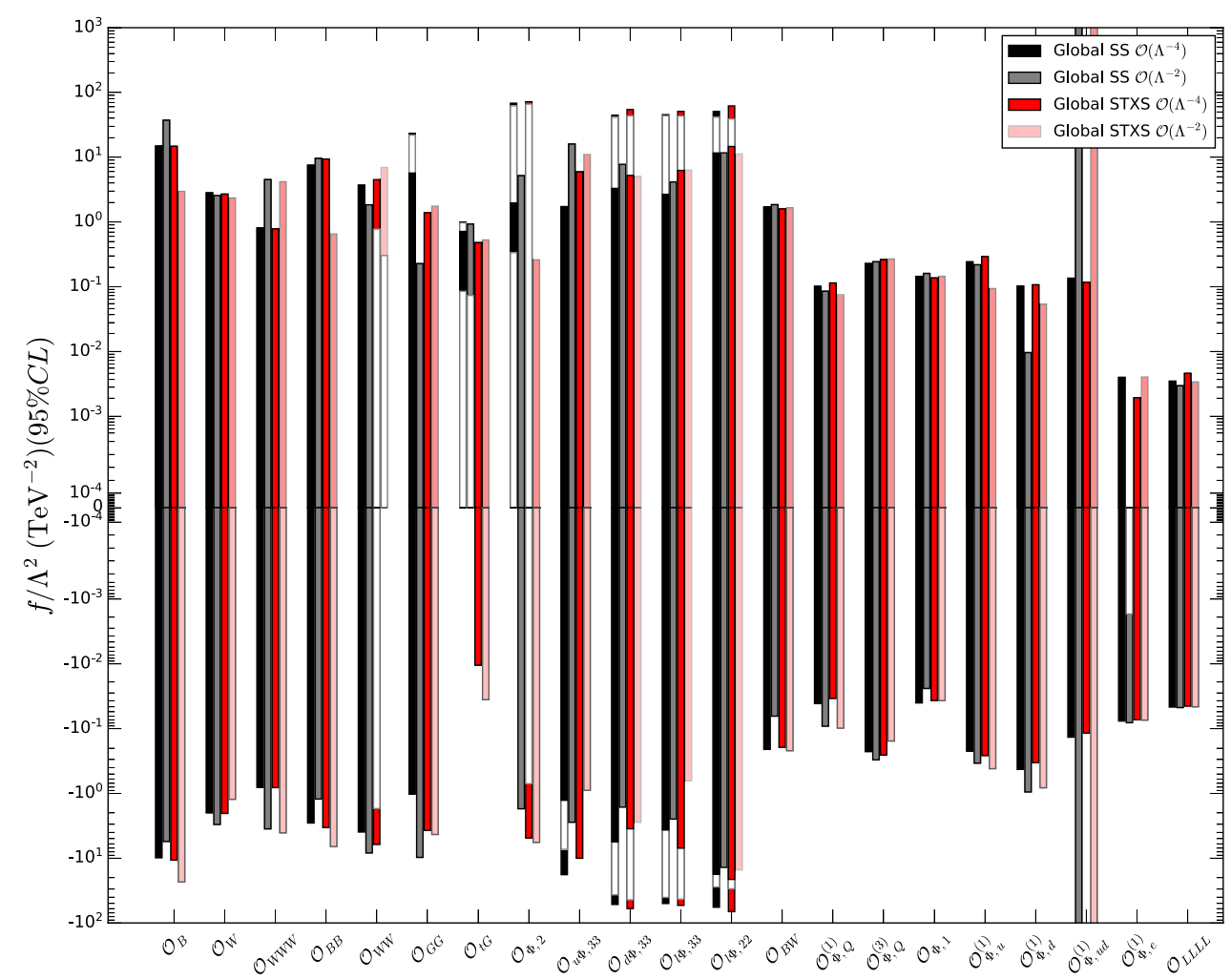

FIG. 12. 95\% C.L. on the 21 Wilson coefficients used in our analyses. The color code indicates the dataset used and the order of the predictions used.

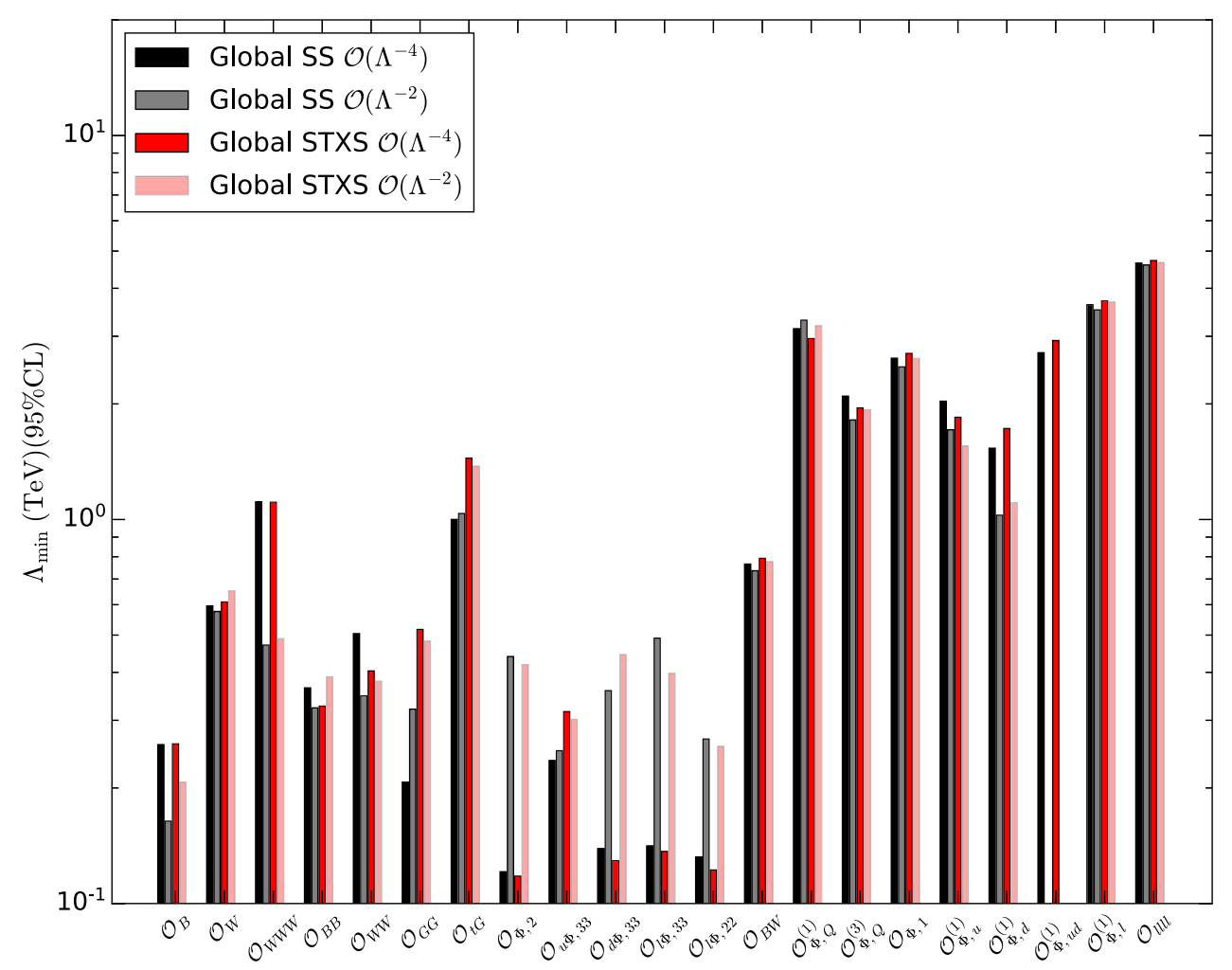

FIG. 13. 95\% C.L. minimal effective scale for each of the 21 Wilson coefficients. The color code indicates the dataset used and the order of the predictions used. 
light quark couplings, but $f_{\Phi, d_{33}}^{(1)}$ is only marginally affected by the inclusion of the EWDBD and Higgs data, and its best fit remains nonzero at $\sim 2 \sigma$ in the global analysis.

(5) The operator $\mathcal{O}_{\Phi, u d}^{(1)}$ induces right-handed chargedcurrent couplings for quarks, and it can only be bound via its quadratic $\left[\mathcal{O}\left(\Lambda^{-4}\right)\right]$ contributions. Including those in the LHC observables, its Wilson coefficient can be bounded with precision comparable to that of the other operators affecting gaugequark couplings; see Fig. 11.

(6) The new EWDBD lead to a significantly improved sensitivity to $\mathcal{O}_{W W W}$ that is the only TGC operator that does not contribute to the Higgs couplings. Furthermore, the constraints on $f_{B} / \Lambda^{2}$ and $f_{W} / \Lambda^{2}$ are also significantly tightened, especially when combining the information from both EWDBD and Higgs data-in particular, the STXS kinematic distributions; see Figs. 3 and 4.

(7) As anticipated, the recently available kinematic distributions of the Higgs not only lead to more stringent limits but also remove some the correlations between the different operators entering into the gluon-gluon-Higgs interactions; see Fig. 6.

(8) Degeneracies associated with the flipping of the sign of some Higgs couplings to gauge bosons and Yukawa couplings, that originate from $\mathcal{O}_{\Phi, 2}$, are not resolved in the present STXS analysis if performed at $\mathcal{O}\left(\Lambda^{-4}\right)$. In fact, we can see clearly the effect of the existence of two $f_{\Phi, 2} / \Lambda^{2}$ local minima in many panels of Fig. 11.

(9) One can check the stability of the results of the overall picture by comparing the bounds derived using just the $1 / \Lambda^{2}$ contribution with those derived with predictions containing terms up to $1 / \Lambda^{4}$; see Fig. 11. From the figure, we read that the analysis performed at $\mathcal{O}\left(1 / \Lambda^{2}\right)$ does not allow for the degenerate solutions associated with the flipping of the sign of the Higgs couplings, or the strong correlations induced in the effective gluon-gluonHiggs interaction or photon-photon-Higgs interaction. Generically, the bounds derived including $1 / \Lambda^{4}$ terms are somewhat stronger. This is particularly the case for the coefficients of $\mathcal{O}_{W W W}$ and $\mathcal{O}_{\Phi d}^{(1)}$, which dominantly contribute to EWDBD. This stability suggests that the power series on $1 / \Lambda$ is under control for the range of energy probed at LHC run 2. Notwithstanding, it is also important to notice that diboson production has serious theoretical problems at $\mathcal{O}\left(\Lambda^{-2}\right)$, since its differential cross section is negative in some regions of the parameter space [108], and the $\mathcal{O}\left(\Lambda^{-4}\right)$ terms should be kept in that case.
(10) As is well known, EFTs have a limited range of validity, which can be signaled, for instance, by the rapid growth of the cross section with the energy and consequent violation of unitarity [35,36]. Consequently, it is a matter of concern whether the bounds on the Wilson coefficients are driven by regions of the phase space where the EFT expansion is no longer valid. Unfortunately, there is no systematic way to truncate the phase space in the observables included in the analysis to avoid such problematic regions. Alternatively, the comparison of the limits obtained by the analyses performed at the orders $1 / \Lambda^{2}$ and $1 / \Lambda^{4}$ allows us to estimate the size of the higher-order contributions that would be most relevant in those phase-space regions. Our results of this comparison indicate that for most operators, the $\mathcal{O}\left(1 / \Lambda^{4}\right)$ contribution is smaller than the leading one, indicating that, generically, the analysis uses EFT in its validity range.

(11) Contrasting with the results of our previous analysis, Refs. [37,109,110], we find that the bounds on bosonic operators modifying the Higgs couplings are much more stringent once the full LHC run 2 data are considered. In particular, the kinematic distributions provided in the STXS format allow the derivation of constraints which are a factor of 2 to 10 stronger for the Wilson coefficients of $\mathcal{O}_{B}, \mathcal{O}_{W}$, $\mathcal{O}_{B B}, \mathcal{O}_{W}$, and $\mathcal{O}_{G G}$ at order $1 / \Lambda^{2}$.

(12) At $\mathcal{O}\left(\Lambda^{-2}\right)$, the precision on the Yukawa couplings $f_{t} / \Lambda^{2}, f_{b} / \Lambda^{2}, f_{\tau} / \Lambda^{2}$, and $f_{\mu} / \Lambda^{2}$ is similar whether using the SS or STXS datasets, and their allowed range is reduced by $\sim 30 \%$ for $f_{b}$ and $f_{\tau}$, and by $\sim 2$ for $f_{\mu}$ with respect to our previous results [37].

(13) Despite the added complexity to the Higgs-gluongluon vertex [see Eq. (2.9) and Fig. 6], the introduction of the additional contribution $\left(\mathcal{O}_{t G}\right)$ to the Higgs production via gluon fusion does not significantly affect the analysis once the independent constraint from top physics [Eq. (3.4)] is included. In addition, the Higgs dataset is able to improve slightly the limits on this coefficient, favoring a value closer to zero, with respect to the top physics bias.

(14) Finally, the study of simplified models shows that the available datasets are able to put stringent limits on the model parameters, as we see in Figs. 9 and 10 and in Eqs. (5.1) and (5.2). In particular, for all 2HMD variants, our analysis results in bounds on $\cos (\beta-\alpha)$, for a fixed $\beta$, which are up to a factor $\sim 4$ stronger than those derived in the experimental analysis of Ref. [106].

Altogether, we find that the increased integrated luminosity gathered at $13 \mathrm{TeV}$ allows us to obtain more stringent bounds on a larger set of anomalous interactions and to perform new tests of the SM. So far, there is no indication of deviations from the SM predictions. 


\section{ACKNOWLEDGMENTS}

This work is supported in part by Conselho Nacional de Desenvolvimento Centífico e Tecnológico (CNPq), Grants No. 307265/2017-0 (A. A.) and No. 305762/2019-2 (O. J. P. E.), and by Fundação de Amparo à Pesquisa do Estado de São Paulo (FAPESP) Grants No. 2018/16921-1 and No. 2019/04837-9. M. C. G.-G. is supported by Spanish Grant No. PID2019-105614GB-C21 financed by MCIN/AEI/10.13039/501100011033, by USA-NSF Grant No. PHY-1915093, and by AGAUR (Generalitat de Catalunya) Grant No. 2017-SGR-929. The authors acknowledge the support of European ITN Grant No. H2020-MSCA-ITN-2019//860881-HIDDeN.
APPENDIX: ANALYTICAL EXPRESSION OF $\chi^{2}$ FOR $\mathcal{O}\left(\Lambda^{-2}\right)$ SS AND STXS ANALYSIS

By definition, when the theoretical predictions for the observables considered in the analysis are expanded to linear order [i.e., $\mathcal{O}\left(\Lambda^{-2}\right)$ ] in the Wilson coefficients, $\Delta \chi^{2}$ takes the form

$$
\Delta \chi^{2}=\sum_{i=1}^{N}\left(\frac{f_{i}}{\Lambda^{2}}-\frac{f_{i}^{0}}{\Lambda^{2}}\right) V_{i j}^{-1}\left(\frac{f_{j}}{\Lambda^{2}}-\frac{f_{j}^{0}}{\Lambda^{2}}\right)
$$

where $V$ is the covariance matrix

$$
V_{i j} \equiv \sigma_{i} \sigma_{j} \rho_{i j}
$$

\begin{tabular}{|c|c|c|c|c|c|c|c|c|c|c|c|c|c|c|c|c|c|c|c|c|}
\hline & $\mathcal{O}_{B}$ & $\mathcal{O}_{W}$ & $V W W$ & $\mathcal{O}_{B B}$ & $W W$ & $G G$ & $\mathcal{O}_{t G}$ & $\mathcal{O}_{\Phi, 2}$ & $\mathcal{O}_{u \Phi, 33}$ & $d \Phi, 33$ & $\mathcal{O}_{e \Phi, 33}$ & $\mathcal{O}_{e \Phi, 2}$ & $\mathcal{O}_{B W}$ & $\mathcal{O}_{\Phi, 1}$ & $\mathcal{O}_{\Phi, Q}^{(1)}$ & $\mathcal{O}_{\Phi, Q}^{(3)}$ & $\mathcal{O}_{\Phi, u}^{(1)}$ & $\mathcal{O}_{\Phi, d}^{(1)}$ & $\mathcal{O}_{\Phi, e}^{(1)}$ & $\mathcal{O}_{\text {LLLLL }}$ \\
\hline 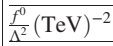 & 16 & 23 & 49 & 4.2 & -3.2 & -4.7 & 0.52 & 1.7 & 6.6 & 3.1 & 0.83 & -1.1 & 0.89 & 0.068 & -0.003 & -0.030 & -0.061 & -0.47 & -0.041 & -( \\
\hline$\sigma$ & 11 & 1.4 & 2.0 & 2.7 & 2.5 & 2.5 & -0.21 & 1.7 & 4.7 & 2.3 & 1.7 & 6. & 0.48 & 0.046 & 0.044 & 0.14 & 0.14 & 0.24 & 0.020 & .013 \\
\hline
\end{tabular}

For the SS analysis, we find the best-fit values and uncertainties

where the correlation matrix in the same order of operators as the above table is

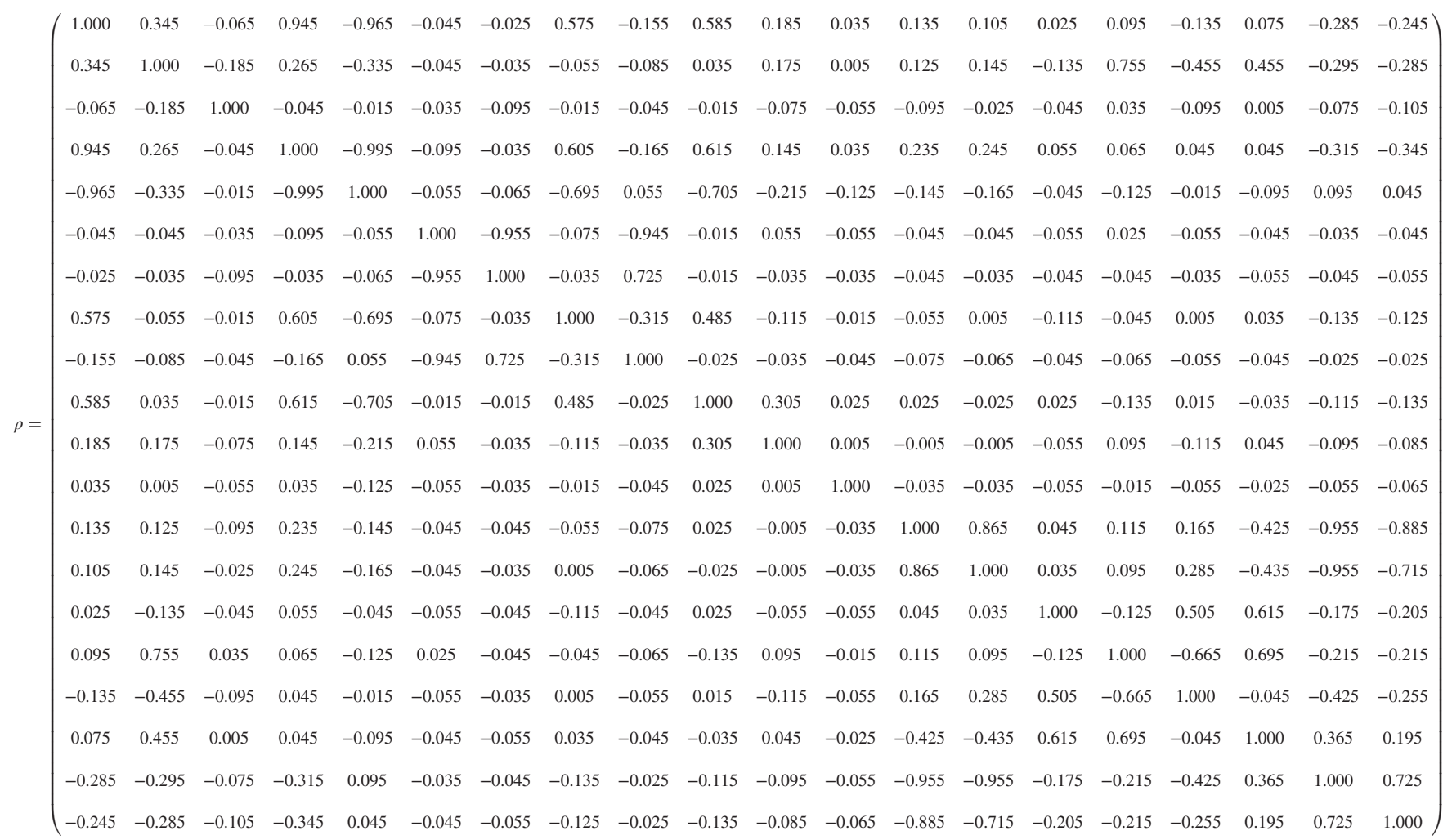

For the STXS analysis, we find the best-fit values and uncertainties

\begin{tabular}{|c|cccccccccccccccccccc|}
\hline & $\mathcal{O}_{B}$ & $\mathcal{O}_{W}$ & $\mathcal{O}_{W W W}$ & $\mathcal{O}_{B B}$ & $\mathcal{O}_{W W}$ & $\mathcal{O}_{G G}$ & $\mathcal{O}_{t G}$ & $\mathcal{O}_{\Phi, 2}$ & $\mathcal{O}_{u \Phi, 33}$ & $\mathcal{O}_{d \Phi, 33}$ & $\mathcal{O}_{e \Phi, 33}$ & $\mathcal{O}_{e \Phi, 22}$ & $\mathcal{O}_{B W}$ & $\mathcal{O}_{\Phi, 1}$ & $\mathcal{O}_{\Phi, Q}^{(1)}$ & $\mathcal{O}_{\Phi, Q}^{(3)}$ & $\mathcal{O}_{\Phi, u}^{(1)}$ & $\mathcal{O}_{\Phi, d}^{(1)}$ & $\mathcal{O}_{\Phi, e}^{(1)}$ & $\mathcal{O}_{L L L L}$ \\
\hline$f^{f^{0}}(\mathrm{TeV})^{-2}$ & -10 & 0.56 & 0.061 & -2.9 & 3.6 & -1.3 & 0.25 & -2.7 & 5.0 & 1.1 & 2.8 & -2.0 & 0.72 & 0.054 & -0.012 & 0.057 & -0.16 & -0.38 & -0.035 & -0.021 \\
\hline$\sigma$ & 6.6 & 0.90 & 2.1 & 1.8 & -1.7 & 1.5 & 0.14 & 1.5 & 3.0 & 2.0 & 1.7 & 6.6 & 0.47 & 0.045 & 0.043 & 0.11 & 0.13 & 0.22 & 0.019 & 0.012 \\
\hline
\end{tabular}

where the correlation matrix in the same order of operators as the above table is 


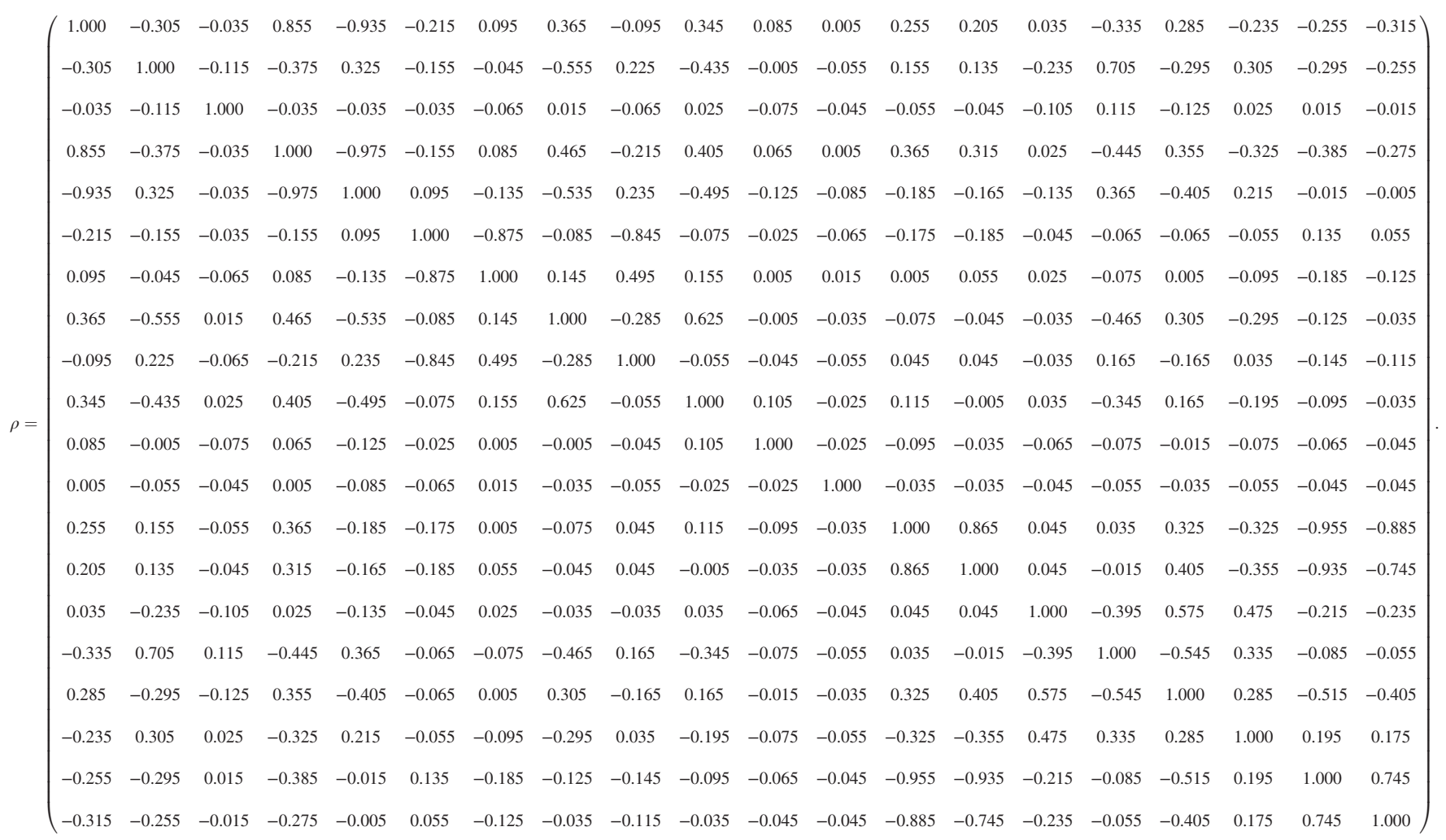

[1] S. Weinberg, Physica (Amsterdam) 96A, 327 (1979).

[2] H. Georgi, Weak Interactions and Modern Particle Theory (Benjamin/Cummings, Menlo Park, 1984), p. 165, ISBN 9780805331639.

[3] J. F. Donoghue, E. Golowich, and B. R. Holstein, Dynamics of the standard model (Cambridge University Press, Cambridge, 1992).

[4] G. Aad et al. (ATLAS Collaboration), Phys. Lett. B 716, 1 (2012).

[5] S. Chatrchyan et al. (CMS Collaboration), Phys. Lett. B 716, 30 (2012).

[6] D. de Florian et al. (LHC Higgs Cross Section Working Group), Report No. CERN-2017-002-M, 2017.

[7] J. R. Andersen et al., in 9th Les Houches Workshop on Physics at TeV Colliders (2016).

[8] A. Falkowski and D. Straub, J. High Energy Phys. 04 (2020) 066.

[9] S. Dawson, S. Homiller, and S. D. Lane, Phys. Rev. D 102, 055012 (2020).

[10] J. De Blas et al., Eur. Phys. J. C 80, 456 (2020).

[11] Anisha, S. Das Bakshi, J. Chakrabortty, and S. K. Patra, Phys. Rev. D 103, 076007 (2021).

[12] J. J. Ethier, G. Magni, F. Maltoni, L. Mantani, E. R. Nocera, J. Rojo, E. Slade, E. Vryonidou, and C. Zhang, J. High Energy Phys. 11 (2021) 089.
[13] J. Ellis, M. Madigan, K. Mimasu, V. Sanz, and T. You, J. High Energy Phys. 04 (2021) 279.

[14] K. Hagiwara, S. Ishihara, R. Szalapski, and D. Zeppenfeld, Phys. Rev. D 48, 2182 (1993).

[15] K. Hagiwara, T. Hatsukano, S. Ishihara, and R. Szalapski, Nucl. Phys. B496, 66 (1997).

[16] B. Grzadkowski, M. Iskrzynski, M. Misiak, and J. Rosiek, J. High Energy Phys. 10 (2010) 085.

[17] S. Weinberg, Phys. Rev. Lett. 43, 1566 (1979).

[18] J. Ellis, V. Sanz, and T. You, J. High Energy Phys. 03 (2015) 157.

[19] A. Falkowski and F. Riva, J. High Energy Phys. 02 (2015) 039.

[20] J. Ellis, C. W. Murphy, V. Sanz, and T. You, J. High Energy Phys. 06 (2018) 146.

[21] E. da Silva Almeida, N. Rosa-Agostinho, O. J. P. Éboli, and M. C. Gonzalez-Garcia, Phys. Rev. D 100, 013003 (2019).

[22] R. Alonso, E. E. Jenkins, A. V. Manohar, and M. Trott, J. High Energy Phys. 04 (2014) 159.

[23] B. Henning, X. Lu, T. Melia, and H. Murayama, J. High Energy Phys. 08 (2017) 016; 09 (2019) 019(E).

[24] T. Corbett, O. J. P. Éboli, J. González-Fraile, and M. C. González-Garcia, Phys. Rev. D 87, 015022 (2013).

[25] H. D. Politzer, Nucl. Phys. B172, 349 (1980).

[26] H. Georgi, Nucl. Phys. B361, 339 (1991). 
[27] C. Arzt, Phys. Lett. B 342, 189 (1995).

[28] H. Simma, Z. Phys. C 61, 67 (1994).

[29] A. De Rujula, M. B. Gavela, P. Hernandez, and E. Masso, Nucl. Phys. B384, 3 (1992).

[30] J. Elias-Miro, J. R. Espinosa, E. Masso, and A. Pomarol, J. High Energy Phys. 11 (2013) 066.

[31] S. Schael et al. (SLD Electroweak Group, DELPHI, ALEPH, SLD, SLD Heavy Flavour Group, OPAL, LEP Electroweak Working Group, L3 Collaborations), Phys. Rep. 427, 257 (2006).

[32] C. Degrande, J. High Energy Phys. 02 (2014) 101.

[33] E. da Silva Almeida, A. Alves, N. Rosa Agostinho, O. J. P. Éboli, and M. C. Gonzalez-Garcia, Phys. Rev. D 99, 033001 (2019).

[34] T. Corbett, O. J.P. Éboli, J. González-Fraile, and M. C. González-Garcia, Phys. Rev. D 86, 075013 (2012).

[35] T. Corbett, O. J. P. Éboli, and M. C. Gonzalez-Garcia, Phys. Rev. D 96, 035006 (2017).

[36] T. Corbett, O. J. P. Éboli, and M. C. Gonzalez-Garcia, Phys. Rev. D 91, 035014 (2015).

[37] A. Alves, N. Rosa-Agostinho, O. J.P. Éboli, and M. C. Gonzalez-Garcia, Phys. Rev. D 98, 013006 (2018).

[38] C. Grojean, E. Salvioni, M. Schlaffer, and A. Weiler, J. High Energy Phys. 05 (2014) 022.

[39] M. Buschmann, C. Englert, D. Goncalves, T. Plehn, and M. Spannowsky, Phys. Rev. D 90, 013010 (2014).

[40] S. Dawson, I. M. Lewis, and M. Zeng, Phys. Rev. D 90, 093007 (2014).

[41] E. E. Jenkins, A. V. Manohar, and M. Trott, J. High Energy Phys. 10 (2013) 087.

[42] E. E. Jenkins, A. V. Manohar, and M. Trott, J. High Energy Phys. 01 (2014) 035.

[43] S. Profumo, M. J. Ramsey-Musolf, C. L. Wainwright, and P. Winslow, Phys. Rev. D 91, 035018 (2015).

[44] C.-Y. Chen, J. Kozaczuk, and I. M. Lewis, J. High Energy Phys. 08 (2017) 096.

[45] I. Brivio, S. Bruggisser, E. Geoffray, W. Kilian, M. Krämer, M. Luchmann, T. Plehn, and B. Summ, arXiv:2108.01094.

[46] S. L. Glashow and S. Weinberg, Phys. Rev. D 15, 1958 (1977).

[47] G. C. Branco, P. M. Ferreira, L. Lavoura, M. N. Rebelo, M. Sher, and J. P. Silva, Phys. Rep. 516, 1 (2012).

[48] M. Gorbahn, J. M. No, and V. Sanz, J. High Energy Phys. 10 (2015) 036.

[49] H. Bélusca-Maçïto, A. Falkowski, D. Fontes, J. C. Romão, and J. A. P. Silva, Eur. Phys. J. C 77, 176 (2017).

[50] D. Alves (LHC New Physics Working Group), J. Phys. G 39, 105005 (2012).

[51] J. de Blas, J. C. Criado, M. Perez-Victoria, and J. Santiago, J. High Energy Phys. 03 (2018) 109.

[52] C. Patrignani et al. (Particle Data Group), Chin. Phys. C 40, 100001 (2016).

[53] ALEPH, CDF, D0, DELPHI, L3, OPAL, SLD, LEP Electroweak Working Group, Tevatron Electroweak Working Group, SLD Electroweak, Heavy Flavour Groups, arXiv:1012.2367.

[54] M. Ciuchini, E. Franco, S. Mishima, M. Pierini, L. Reina, and L. Silvestrini, Nucl. Part. Phys. Proc. 273, 2219 (2016).
[55] The LEP, ALEPH, DELPHI, L3, OPAL, and the LEP TGC Working Group Collaborations, A Combination of Preliminary Results on Gauge Boson Couplings Measured by the LEP Experiments, http://lepewwg.web.cern.ch/ LEPEWWG/lepww/tgc.

[56] G. Aad et al. (ATLAS Collaboration), J. High Energy Phys. 09 (2016) 029.

[57] V. Khachatryan et al. (CMS Collaboration), Eur. Phys. J. C 76, 401 (2016).

[58] G. Aad et al. (ATLAS Collaboration), Phys. Rev. D 93, 092004 (2016).

[59] V. Khachatryan et al. (CMS Collaboration), Eur. Phys. J. C 77, 236 (2017).

[60] ATLAS Collaboration, Report No. ATLAS-CONF-2016043, 2016, https://cds.cern.ch/record/2206093.

[61] ATLAS Collaboration, Report No. ATLAS-CONF-2018034, 2018, https://cds.cern.ch/record/2630187.

[62] M. Aaboud et al. (ATLAS Collaboration), Eur. Phys. J. C 77, 563 (2017).

[63] CMS Collaboration, Report No. CMS-PAS-SMP-20-014, 2021, https://cds.cern.ch/record/2758362.

[64] A. M. Sirunyan et al. (CMS Collaboration), Phys. Rev. D 102, 092001 (2020).

[65] CMS Collaboration, Report No. CMS-PAS-SMP-20-005, 2021, https://cds.cern.ch/record/2757267.

[66] M. Aaboud et al. (ATLAS Collaboration), Eur. Phys. J. C 78, 24 (2018).

[67] G. Aad et al. (ATLAS Collaboration), Eur. Phys. J. C 81, 163 (2021).

[68] R. Frederix, S. Frixione, V. Hirschi, D. Pagani, H. S. Shao, and M. Zaro, J. High Energy Phys. 07 (2018) 185.

[69] N. D. Christensen and C. Duhr, Comput. Phys. Commun. 180, 1614 (2009).

[70] A. Alloul, N. D. Christensen, C. Degrande, C. Duhr, and B. Fuks, Comput. Phys. Commun. 185, 2250 (2014).

[71] T. Sjostrand, S. Mrenna, and P. Z. Skands, Comput. Phys. Commun. 178, 852 (2008).

[72] J. de Favereau, C. Delaere, P. Demin, A. Giammanco, V. Lemaitre, A. Mertens, and M. Selvaggi (DELPHES 3 Collaboration), J. High Energy Phys. 02 (2014) 057.

[73] M. Cacciari, G. P. Salam, and G. Soyez, Eur. Phys. J. C 72, 1896 (2012).

[74] ATLAS Collaboration, Report No. ATLAS-CONF-2020053, 2020, https://cds.cern.ch/record/2743067.

[75] ATLAS Collaboration, Report No. ATLAS-CONF-2020026, 2020, https://cds.cern.ch/record/2725727.

[76] G. Aad et al. (ATLAS Collaboration), Eur. Phys. J. C 80, 957 (2020); 81, 29(E) (2021); 81, 398(E) (2021).

[77] G. Aad et al. (ATLAS Collaboration), Eur. Phys. J. C 81, 178 (2021).

[78] G. Aad et al. (ATLAS, CMS Collaborations), J. High Energy Phys. 08 (2016) 045.

[79] G. Aad et al. (ATLAS Collaboration), Eur. Phys. J. C 76, 6 (2016).

[80] ATLAS Collaboration, Report No. ATLAS-CONF-2020027, 2020, https://cds.cern.ch/record/2725733.

[81] G. Aad et al. (ATLAS Collaboration), Phys. Lett. B 809, 135754 (2020).

[82] G. Aad et al. (ATLAS Collaboration), Phys. Lett. B 812, 135980 (2021). 
[83] CMS Collaboration, Report No. CMS-PAS-HIG-19-005, 2020, https://cds.cern.ch/record/2706103.

[84] CMS Collaboration, Report No. CMS-PAS-HIG-19-015, 2020, https://cds.cern.ch/record/2725142.

[85] A. M. Sirunyan et al. (CMS Collaboration), Eur. Phys. J. C 81, 488 (2021).

[86] CMS Collaboration, Report No. CMS-PAS-HIG-19-010, 2020, https://cds.cern.ch/record/2725590.

[87] CMS Collaboration, Report No. CMS-PAS-HIG-19-017, 2021, https://cds.cern.ch/record/2758367.

[88] V. Hirschi and O. Mattelaer, J. High Energy Phys. 10 (2015) 146.

[89] C. Degrande, G. Durieux, F. Maltoni, K. Mimasu, E. Vryonidou, and C. Zhang, Phys. Rev. D 103, 096024 (2021).

[90] A. Buckley, J. Butterworth, D. Grellscheid, H. Hoeth, L. Lonnblad, J. Monk, H. Schulz, and F. Siegert, Comput. Phys. Commun. 184, 2803 (2013).

[91] N. Deutschmann, C. Duhr, F. Maltoni, and E. Vryonidou, J. High Energy Phys. 12 (2017) 063; 02 (2018) 159(E).

[92] M. Grazzini, A. Ilnicka, and M. Spira, Eur. Phys. J. C 78, 808 (2018).

[93] A. Buckley, C. Englert, J. Ferrando, D. J. Miller, L. Moore, M. Russell, and C. D. White, J. High Energy Phys. 04 (2016) 015.

[94] D. Barducci et al., arXiv:1802.07237.

[95] I. Brivio, S. Bruggisser, F. Maltoni, R. Moutafis, T. Plehn, E. Vryonidou, S. Westhoff, and C. Zhang, J. High Energy Phys. 02 (2020) 131.

[96] A. M. Sirunyan et al. (CMS Collaboration), Eur. Phys. J. C 79, 886 (2019).
[97] S. Bißmann, C. Grunwald, G. Hiller, and K. Kröninger, J. High Energy Phys. 06 (2021) 010.

[98] T. Corbett, O. J.P. Éboli, J. González-Fraile, and M. C. González-Garcia, Phys. Rev. Lett. 111, 011801 (2013).

[99] A. Azatov, J. Elias-Miro, Y. Reyimuaji, and E. Venturini, J. High Energy Phys. 10 (2017) 027.

[100] A. Azatov, D. Barducci, and E. Venturini, J. High Energy Phys. 04 (2019) 075.

[101] V. Barger, M. McCaskey, and G. Shaughnessy, Phys. Rev. D 81, 034020 (2010).

[102] S. Biswas, E. Gabrielli, F. Margaroli, and B. Mele, J. High Energy Phys. 07 (2013) 073.

[103] J. Chang, K. Cheung, J. S. Lee, and C.-T. Lu, J. High Energy Phys. 05 (2014) 062.

[104] A. Biekoetter, T. Corbett, and T. Plehn, SciPost Phys. 6, 064 (2019).

[105] J. Brehmer, S. Dawson, S. Homiller, F. Kling, and T. Plehn, J. High Energy Phys. 11 (2019) 034.

[106] A. M. Sirunyan et al. (CMS Collaboration), Eur. Phys. J. C 79, 421 (2019).

[107] C. K. Khosa and V. Sanz, arXiv:2102.13429.

[108] J. Baglio, S. Dawson, and I. M. Lewis, Phys. Rev. D 96, 073003 (2017).

[109] T. Corbett, O. J. P. Éboli, D. Goncalves, J. González-Fraile, T. Plehn, and M. Rauch, J. High Energy Phys. 08 (2015) 156.

[110] A. Butter, O. J. P. Éboli, J. Gonzalez-Fraile, M. C. GonzalezGarcia, T. Plehn, and M. Rauch, J. High Energy Phys. 07 (2016) 152. 